\title{
Quantum oscillations and Dirac-Landau levels in Weyl superconductors
}

\author{
Tianyu Liu, ${ }^{1,2,3}$ M. Franz, ${ }^{1,2}$ and Satoshi Fujimoto ${ }^{3}$ \\ ${ }^{1}$ Department of Physics and Astronomy, University of British Columbia, Vancouver, BC, Canada V6T $1 Z 1$ \\ ${ }^{2}$ Quantum Matter Institute, University of British Columbia, Vancouver BC, Canada V6T 1Z4 \\ ${ }^{3}$ Department of Materials Engineering Science, Osaka University, Toyonaka 560-8531, Japan
}

(Dated: January 10, 2018)

\begin{abstract}
When magnetic field is applied to metals and semimetals quantum oscillations appear as individual Landau levels cross the Fermi level. Quantum oscillations generally do not occur in superconductors (SC) because magnetic field is either expelled from the sample interior or, if strong enough, drives the material into the normal state. In addition, elementary excitations of a superconductor - Bogoliubov quasiparticles - do not carry a well defined electric charge and therefore do not couple in a simple way to the applied magnetic field. We predict here that in Weyl superconductors certain types of elastic strain have the ability to induce chiral pseudo-magnetic field which can reorganize the electronic states into Dirac-Landau levels with linear band crossings at low energy. The resulting quantum oscillations in the quasiparticle density of states and thermal conductivity can be experimentally observed under a bending deformation of a thin film Weyl SC and provide new insights into this fascinating family of materials.
\end{abstract}

\section{INTRODUCTION}

Quantum oscillations [1 furnish an essential experimental tool for measuring the Fermi surface of metals. They also help to understand electronic structures of the recently discovered topological insulators [2, 6] and topological Dirac and Weyl semimetals [7-10]. However, probing superconductors by the quantum oscillation technique has been thought impossible because such measurements require strong magnetic fields which are either expelled from the SC due to the Meissner effect or render the material normal. Type-II superconductors allow the field to penetrate but form the Abrikosov vortex state, whose quasiparticle eigenstates are known to be Bloch waves and not Landau levels [11 13 .

Quantum oscillations in resistivity [14 16, Hall coefficient 17, thermal conductivity 18, and torque 19 have already been observed in underdoped cuprates when magnetic field suppresses superconductivity. Quantum oscillations with $1 / \sqrt{B}$ periodicity have also been predicted to appear in vortex lattice 20 and vortex liquid states [21] in cuprates and are observed in $2 \mathrm{H}-\mathrm{NbSe}_{2}[22$. However, reports on conventional quantum oscillations periodic in $1 / B$ in the superconducting state are lacking presumably due to the reasons listed above.

We argue here that this difficulty can be overcome by using the recently proposed Dirac and Weyl superconductors 23, 24, which possess unusual electronic structures comprising linearly dispersing quasiparticle bands at low energies, similar to graphene and $d$-wave $\mathrm{SC}$ in two dimensions and to Dirac and Weyl semimetals in three dimensions. One may expect that Dirac and Weyl superconductors will exhibit a variety of exotic behaviors similar to their semimetal counterparts and to $d$-wave SC. Specifically, it has long been known that elastic strain can induce chiral pseudo-magnetic field and Landau quantization in graphene [25, 26]. Similar effects have been predicted to occur in 3D Dirac and Weyl semimetals [27 33], and very recently also in $d$-wave SC 34,35 .
In this work, through a combination of analytical calculations and numerical simulations, we demonstrate that quantum oscillations can also occur in Dirac and Weyl superconductors under certain types of elastic deformations at zero magnetic field. Remarkably, these quantum oscillations arise due to the formation of Landau levels comprised of charge neutral Bogoliubov quasiparticles deep in the superconducting state. To support these findings we organize the paper as follows. In Section [II] we formulate a model of a Weyl superconductor and discuss its spectrum and phase diagram. In Section [II], we incorporate strain to our Hamiltonian and show that to leading order it produces pseudo-magnetic field in the low-energy sector. In Section [IV] we show that the strain-induced pseudo-magnetic field can give rise to quantum oscillations in density of states (DOS) and longitudinal thermal conductivity. Section $\mathrm{V}$ concludes the paper by discussing the experimental feasibility in candidate materials and outlines various potentially interesting directions based on our current work.

\section{MODEL HAMILTONIAN}

We employ the multilayer model of Meng and Balents 24] as illustrated in Fig. 1. The model comprises alternating topological insulator (TI) and $s$-wave SC layers stacked along the $z$-direction. For the TI layers, for simplicity, only the surface states are considered. In the following we modify the Meng-Balents model slightly by adding anisotropy to the Zeeman mass term, which will allow us regularize the Hamiltonian on the tight binding lattice without adding extra Weyl points near the corners of the Brillouin zone.

The Hamiltonian of such a TI-SC multilayer system reads

$$
H=H_{\mathrm{TI}}+H_{\mathrm{SC}}+H_{t_{d}}+H_{t_{s}}
$$


(a)

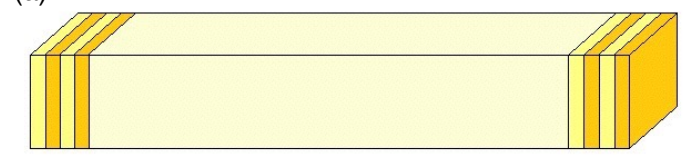

(b)

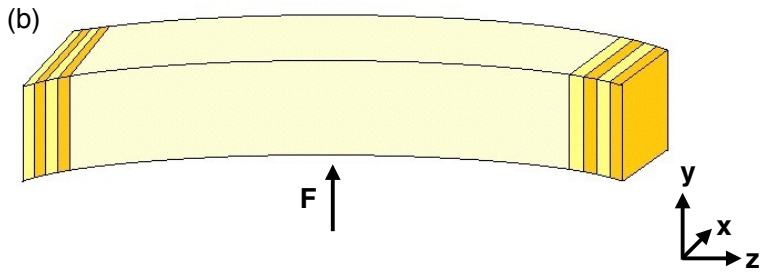

FIG. 1: Schematic plot for (a) undeformed and (b) bent TISC multilayer Weyl superconductor. The alternating TI and SC layers are omitted in the bulk but explicitly drawn at ends to illustrate that there are integer number of unit cells comprised of one TI layer and one SC layer.

where

$$
\begin{gathered}
H_{\mathrm{TI}}=\sum_{\boldsymbol{k}_{\perp}, z} \psi_{\boldsymbol{k}_{\perp} z}^{\dagger}\left[\hbar v_{F} \sigma^{z}(\hat{z} \times \boldsymbol{s}) \cdot \boldsymbol{k}_{\perp}+\left(m-m^{\prime} a^{2} k_{\perp}^{2}\right) s^{z}\right] \psi_{\boldsymbol{k}_{\perp}} \\
H_{\mathrm{SC}}=\sum_{\boldsymbol{k}_{\perp}, z}\left(\Delta c_{\boldsymbol{k}_{\perp} z, 1 \uparrow}^{\dagger} c_{-\boldsymbol{k}_{\perp} z, 1 \downarrow}^{\dagger}+\Delta c_{\boldsymbol{k}_{\perp} z, 2 \uparrow}^{\dagger} c_{-\boldsymbol{k}_{\perp} z, 2 \downarrow}^{\dagger}\right)+\text { h.c. } \\
H_{t_{d}}=\sum_{\boldsymbol{k}_{\perp}, z}\left(\frac{1}{2} t_{d} \psi_{\boldsymbol{k}_{\perp} z+1}^{\dagger} \sigma^{+} \psi_{\boldsymbol{k}_{\perp} z}+\frac{1}{2} t_{d} \psi_{\boldsymbol{k}_{\perp} z-1}^{\dagger} \sigma^{-} \psi_{\boldsymbol{k}_{\perp} z}\right) \\
H_{t_{s}}=\sum_{\boldsymbol{k}_{\perp}, z} \psi_{\boldsymbol{k}_{\perp} z}^{\dagger} t_{s} \sigma^{x} \psi_{\boldsymbol{k}_{\perp} z}
\end{gathered}
$$

The basis $\psi_{\boldsymbol{k}_{\perp} z}=\left(c_{\boldsymbol{k}_{\perp} z, 1 \uparrow}, c_{\boldsymbol{k}_{\perp} z, 1 \downarrow}, c_{\boldsymbol{k}_{\perp} z, 2 \uparrow}, c_{\boldsymbol{k}_{\perp} z, 2 \downarrow}\right)^{T}$ is written in terms of annihilation operators $c_{\boldsymbol{k}_{\perp} z, \sigma s}$ for electrons located in the $z$-th unit cell with an in-plane momentum $\boldsymbol{k}_{\perp}=\left(k_{x}, k_{y}\right)$ and spin projection $s_{z}=\uparrow, \downarrow$. "Sublattice" labels $\sigma_{z}=1,2$ specify the TI-SC interface in the single unit cell. Pauli matrices $\boldsymbol{s}$ and $\boldsymbol{\sigma}$ act in spin and sublattice space, respectively. Physically, $H_{\mathrm{TI}}, H_{\mathrm{SC}}$, $H_{t_{d}}$, and $H_{t_{s}}$ can be interpreted as describing the Zeeman gapped topological insulator surface states, proximityinduced pairing, hopping between adjacent unit cells, and hopping within a single unit cell, respectively. The $\mathrm{m}^{\prime}$ term in $H_{\mathrm{TI}}$ represents the above mentioned modification of the Meng-Balents model (it is easy to check that it has no significant effect at small $k$ as long as $m^{\prime}$ is chosen appropriately small).

As wtitten Hamiltonian Eq. (1) is $\boldsymbol{k} \cdot \boldsymbol{p}$ in $x$-y plane and tight-binding in $z$-direction. It will be useful to apply lattice regularization. We use a simple cubic lattice with lattice constant $a$ and replace $k_{x, y} \rightarrow \frac{1}{a} \sin a k_{x, y}$ and $k_{x, y}^{2} \rightarrow \frac{2}{a^{2}}\left(1-\cos a k_{x, y}\right)$. After partial Fourier transform in the $z$-direction, the Hamiltonian can be written as

$$
H=\frac{1}{2} \sum_{k} \Psi_{k}^{\dagger} \mathcal{H}_{k} \Psi_{k}
$$

with $\Psi_{\boldsymbol{k}}=\left(c_{\boldsymbol{k}, 1 \uparrow}, c_{\boldsymbol{k}, 1 \downarrow}, c_{\boldsymbol{k}, 2 \uparrow}, c_{\boldsymbol{k}, 2 \downarrow}, c_{-\boldsymbol{k}, 1 \uparrow}^{\dagger}, c_{-\boldsymbol{k}, 1 \downarrow}^{\dagger}, c_{-\boldsymbol{k}, 2 \uparrow}^{\dagger}, c_{-\boldsymbol{k}, 2 \downarrow}^{\dagger}\right)^{T}$ and

$$
\begin{gathered}
\mathcal{H}_{\boldsymbol{k}}=\left(m-4 m^{\prime}+2 m^{\prime} \cos k_{x} a+2 m^{\prime} \cos k_{y} a\right) s^{z} \tau^{z}+ \\
t_{d} \sin k_{z} a \sigma^{y} \tau^{z}+\left(t_{s}+t_{d} \cos k_{z} a\right) \sigma^{x} \tau^{z}+\frac{\hbar v_{F}}{a} \sin k_{y} a s^{x} \sigma^{z} \\
-\frac{\hbar v_{F}}{a} \sin k_{x} a s^{y} \sigma^{z} \tau^{z}-\operatorname{Im} \Delta s^{y} \tau^{x}-\operatorname{Re} \Delta s^{y} \tau^{y}
\end{gathered}
$$

The spectrum of $\mathcal{H}_{\boldsymbol{k}}$ reads

$$
\begin{aligned}
\epsilon_{\boldsymbol{k}, \pm}^{2}= & \frac{\hbar^{2} v_{F}^{2}}{a^{2}}\left(\sin ^{2} k_{x} a+\sin ^{2} k_{y} a\right)+ \\
& \left(m-4 m^{\prime}+2 m^{\prime} \cos k_{x} a+2 m^{\prime} \cos k_{y} a\right. \\
& \left. \pm \sqrt{t_{s}^{2}+t_{d}^{2}+2 t_{s} t_{d} \cos k_{z} a+|\Delta|^{2}}\right)^{2}
\end{aligned}
$$

We plot the spectrum in Fig. 2 for a system with $\bar{l}_{y}=$ 500 layers and open boundary conditions along the $y$ direction and periodic boundary conditions in the other two dimensions. We set $\Delta=1$ and measure all other parameters in terms of $\Delta$. We take $m=10.26, m^{\prime}=$ $2.53, \hbar v_{F} / a=1, t_{d}=-4.79, t_{s}=14.86$, and the lattice constant is set to be $a=6 \AA$. These values will also be used in our numerical simulations unless other values are specified.

Without loss of generality, we have assumed $m, m^{\prime}>0$ in the following discussion. Thus, the sector $\epsilon_{\boldsymbol{k},+}$ is fully gapped while $\epsilon_{\boldsymbol{k},-}$ can be gapless when

$$
\sqrt{\left(\left|t_{s}\right|-\left|t_{d}\right|\right)^{2}+|\Delta|^{2}}<m<\sqrt{\left(\left|t_{s}\right|+\left|t_{d}\right|\right)^{2}+|\Delta|^{2}}
$$

If Eq. (5) holds, nondegenerate quasiparticle bands exhibit a point node at $\boldsymbol{k}_{W}=(0,0, \eta Q)$ with

$$
Q a=\cos ^{-1}\left(\frac{m^{2}-t_{s}^{2}-t_{d}^{2}-|\Delta|^{2}}{2 t_{s} t_{d}}\right)
$$

and $\eta= \pm 1$. As expected, the system is a Weyl superconductor.

To understand the low-energy physics better, we introduce $2 \times 2$ auxiliary matrices

$$
\begin{aligned}
& D_{\boldsymbol{k}, \pm}=\frac{\hbar v_{F}}{a} \sin k_{y} a \kappa^{x}-\frac{\hbar v_{F}}{a} \sin k_{x} a \kappa^{y}+ \\
& \left(M_{k_{z}, \pm}-4 m^{\prime}+2 m^{\prime} \cos k_{x} a+2 m^{\prime} \cos k_{y} a\right) \kappa^{z}
\end{aligned}
$$

where $\boldsymbol{\kappa}$ are Pauli matrices in transformed Nambu space and

$$
M_{k_{z}, \pm}=m \pm \sqrt{t_{s}^{2}+t_{d}^{2}+2 t_{s} t_{d} \cos k_{z} a+|\Delta|^{2}}
$$

As $\epsilon_{\boldsymbol{k}, \pm}$ is also the dispersion for $D_{\boldsymbol{k}, \pm}$, the low-energy physics of Eq. (3) may be understood by studying $D_{\boldsymbol{k},-}$ because there always exists a unitary transformation $U$ that can block diagonalize $\mathcal{H}_{\boldsymbol{k}}$

$$
U^{-1} \mathcal{H}_{\boldsymbol{k}} U=\operatorname{diag}\left(D_{\boldsymbol{k},-}, D_{\boldsymbol{k},-}, D_{\boldsymbol{k},+}, D_{\boldsymbol{k},+}\right)
$$



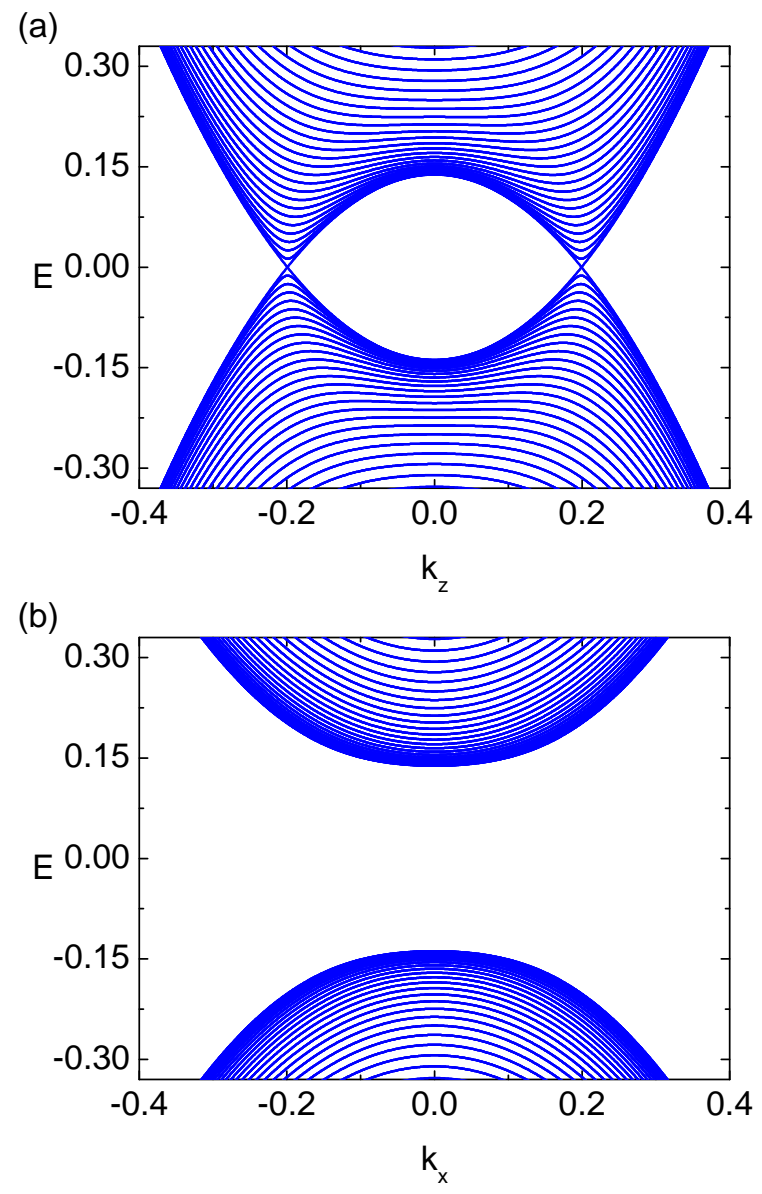

FIG. 2: Band structure of a Weyl superconductor plotted (a) along $k_{z}$-axis with $k_{x}=0$ and (b) along $k_{x}$-axis with $k_{z}=0$. Periodic boundary conditions are applied in $x, z$ directions while the system is chosen to have $\bar{l}_{y}=500$ layers in $y$-direction. The parameters are listed below Eq. (4).

For fixed $k_{z}$ value, we rewrite $D_{\boldsymbol{k},-}$ in a $\boldsymbol{k} \cdot \boldsymbol{p}$ fashion,

$$
D_{\boldsymbol{k} \cdot \boldsymbol{p}}^{-}=\left(\begin{array}{cc}
-m^{\prime} a^{2}\left(k_{x}^{2}+k_{y}^{2}\right)+\mu_{\mathrm{eff}} & i \hbar v_{F}\left(k_{x}-i k_{y}\right) \\
-i \hbar v_{F}\left(k_{x}+i k_{y}\right) & m^{\prime} a^{2}\left(k_{x}^{2}+k_{y}^{2}\right)-\mu_{\mathrm{eff}}
\end{array}\right)
$$

We notice that $D_{\boldsymbol{k} \cdot \boldsymbol{p}}^{-}$can be regarded as describing a $p_{x}+$ $i p_{y}$ superconductor with an effective chemical potential $\mu_{\text {eff }}=M_{k_{z},-}$. It is characterized by Chern number

$$
C=\frac{1}{2}\left[\operatorname{sgn}\left(\mu_{\mathrm{eff}}\right)+\operatorname{sgn}\left(m^{\prime}\right)\right] .
$$

If Eq. (5) holds, for those $k_{z}$ 's that satisfy $\mu_{\text {eff }}=M_{k_{z},-}>$ 0 , this $\mathrm{SC}$ is in the weak pairing phase with Chern number $C=1$. As a result, for each of such $k_{z}$ 's, there exist counter propagating chiral Majorana states on boundaries open in the $y$-direction. Therefore, the edge states of Eq. (1) are Majorana-Fermi arcs as illustrated in Fig. 3(a).

To understand the phases of this model consider a value of $m$ that satisfies Eq. (5) with fixed $\Delta$. Now increase it such that $m>\sqrt{\left(\left|t_{s}\right|+\left|t_{d}\right|\right)^{2}+|\Delta|^{2}}$; according to Eq. (4), our Weyl superconductor will be gapped into a topologically superconducting phase, whose spectrum is shown in Fig. 3(b). It exhibits a surface mode because the Chern number is still $C=1$ for all $k_{z}$. On the other hand, if $m$ is decreased to $m<\sqrt{\left(\left|t_{s}\right|-\left|t_{d}\right|\right)^{2}+|\Delta|^{2}}$, the system enters a trivial superconducting phase with no edge modes, as shown in Fig. 3(c). If $m$ is fixed to a value obeying Eq. (5), but $|\Delta|$ is gradually increased, eventually, $m$ is overwhelmed by $\sqrt{\left(\left|t_{s}\right|-\left|t_{d}\right|\right)^{2}+|\Delta|^{2}}$ and the system becomes a trivial superconductor, as indicated by Fig. 3(d).

Based on the above considerations, we plot the global phase diagram of our Weyl superconductor in $|\Delta|-m$ plane in Fig. 4. The phase boundaries are given by two hyperbolas,

$$
\begin{aligned}
& m^{2}-|\Delta|^{2}=\left(\left|t_{s}\right|+\left|t_{d}\right|\right)^{2}, \\
& m^{2}-|\Delta|^{2}=\left(\left|t_{s}\right|-\left|t_{d}\right|\right)^{2} .
\end{aligned}
$$

Above the upper bound Eq. (10), the multilayer is a topological superconductor, which can be viewed as a stack of $2 \mathrm{D} p_{x}+i p_{y}$ superconductors. These are known to posess counter propagating chiral Majorana edge modes on a pair of parallel boundaries. Since switching off the superconductivity will give a 3D quantum anomalous Hall (QAH) insulator [36], the multilayer topological superconductor structure may be refered to as "3D QAH superconductor". Below the lower bound Eq. (11), the multilayer is a trivial superconductor while between them it is a Weyl superconductor.

\section{STRAIN-INDUCED GAUGE FIELD}

In the preceding section, we studied electronic structure and phase diagram of multilayer model of Weyl superconductor. In this section, we will understand how the electronic structure is changed under generic strain.

When elastic strain distorts the lattice, the chemical bonds are stretched and compressed. Orbital orientations are also rotated, making the symmetry-prohibited hoppings now non-zero. For our purposes, the most important modification comes from the replacement of hopping amplitudes along $z$-direction [29, 33, 37.

$$
\begin{gathered}
t_{d} \sigma^{ \pm} \rightarrow t_{d}\left(1-w_{33}\right) \sigma^{ \pm}-i \frac{\hbar v_{F}}{a} w_{31} s^{y} \sigma^{z}+i \frac{\hbar v_{F}}{a} w_{32} s^{x} \sigma^{z} \\
t_{s} \rightarrow t_{s}\left(1-w_{33}\right)
\end{gathered}
$$

with the strain tensor $w_{i j}=\frac{1}{2}\left(\partial_{i} u_{j}+\partial_{j} u_{i}\right)$, where $u_{j}$ is the $j$-th component of the displacement vector $\boldsymbol{u}$. Under such hopping parameter substitution, the Hamiltonian in Eq. (3) is changed to

$$
\tilde{\mathcal{H}}_{k}=\mathcal{H}_{k}+\delta \mathcal{H}_{k}
$$


(a)

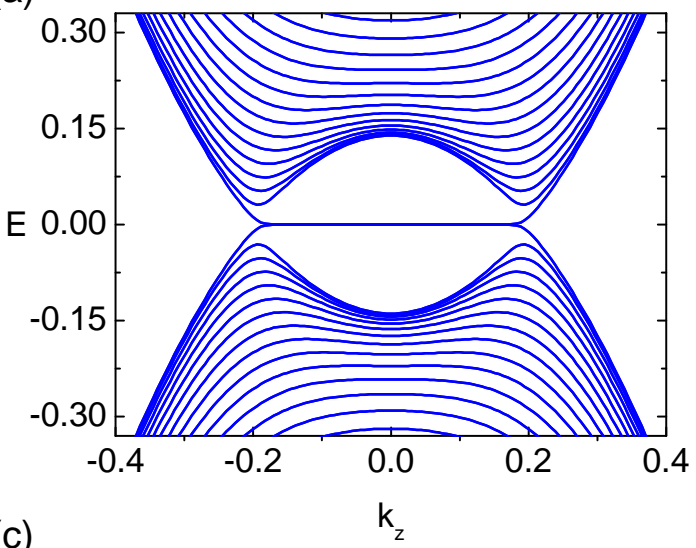

(c)

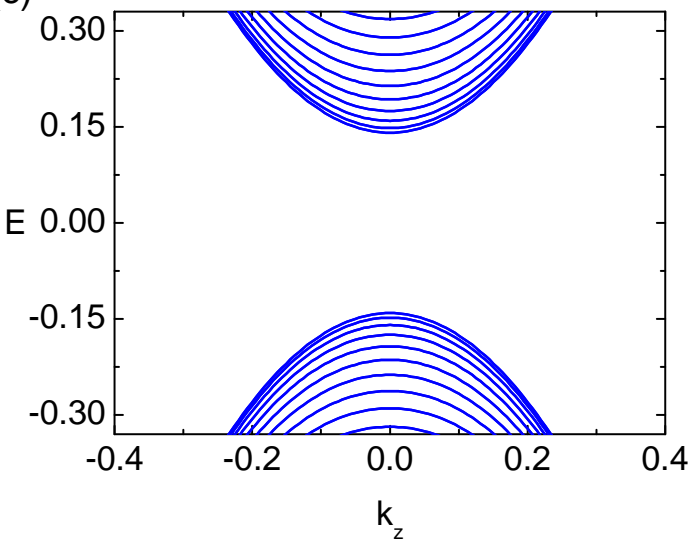

(b)

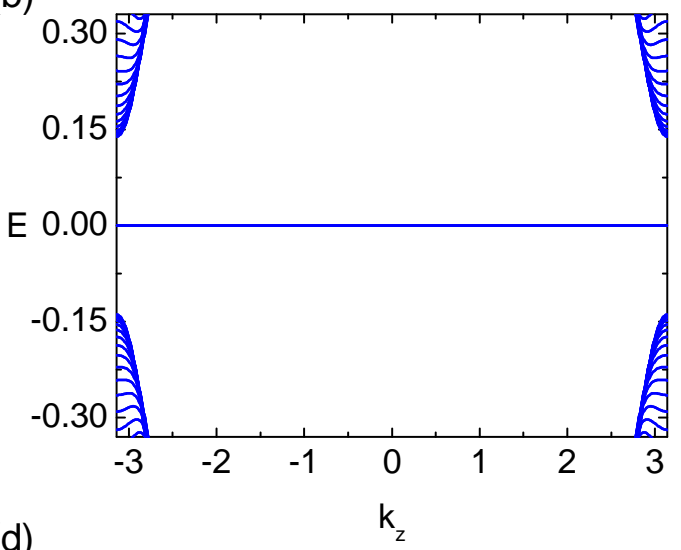

(d)

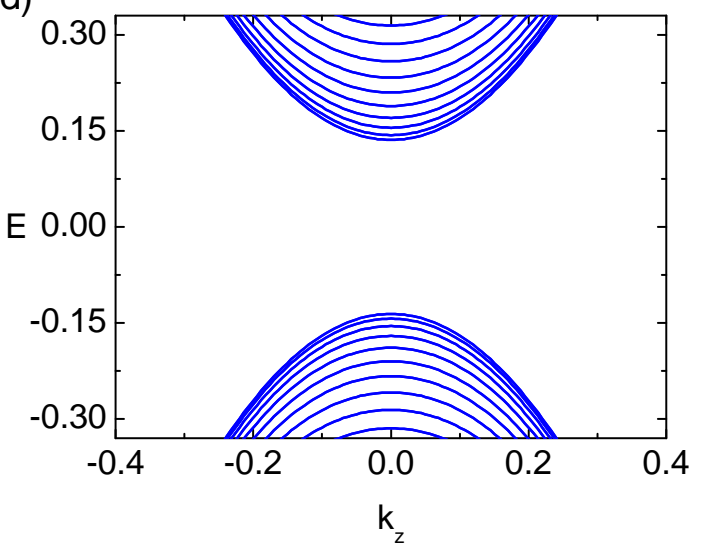

FIG. 3: Band structure of a Weyl superconductor with open boundary conditions and $\bar{l}_{y}=150$ layers along the $y$-direction. All panels are plotted along $k_{z}$-axis with $k_{x}=0$ and with patameters as in Fig. 2 . (a) Weyl superconductor phase for $(m, \Delta)=(10.26,1)$. A Fermi arc connecting two Weyl points appears due to the chiral Majorana edge states of the effective $p_{x}+i p_{y}$ superconductors that emerge for fixed $k_{z}$ between the Weyl nodes. (b) Topological superconductor phase for $(m, \Delta)=$ $(19.82,1)$. The increase of $m$ will separate two Weyl points and extend Fermi arc. When two Weyl points meet at Brillouin zone boundary, they annihilate and open up a SC gap but leave behind the Fermi arc extended over the whole BZ. (c) Trivial superconductor phase for $(m, \Delta)=(9.98,1)$. The decrease of $m$ makes two Weyl points meet at Brillouin zone center and annihilation and leads to the disappearance of the Fermi arc. (d) Trivial superconductor phase with $(m, \Delta)=(10.26,2.56)$. The increase of $\Delta$ is equivalent to decrease of $m$ and Weyl points again annihilate at the BZ center.

where the correction due to strain is

$$
\begin{aligned}
& \delta \mathcal{H}_{\boldsymbol{k}}=-\left(t_{s} w_{33}+t_{d} w_{33} \cos k_{z} a\right) \sigma^{x} \tau^{z}-t_{d} w_{33} \sigma^{y} \tau^{z} \sin k_{z} a \\
& -\frac{\hbar v_{F}}{a} w_{31} s^{y} \sigma^{z} \tau^{z} \sin k_{z} a+\frac{\hbar v_{F}}{a} w_{32} s^{x} \sigma^{z} \sin k_{z} a
\end{aligned}
$$

To understand the effect of strain on the low-energy physics we consider $\tilde{\mathcal{H}}_{k}$ in the vicinity of Weyl points $\boldsymbol{k}_{W}=(0,0, \eta Q)$ and expand

$$
\tilde{\mathcal{H}}_{\boldsymbol{k}_{W}+\boldsymbol{q}}=\mathcal{H}_{\boldsymbol{k}_{W}}+h_{\boldsymbol{q}}+\delta \mathcal{H}_{\boldsymbol{k}_{W}}+O\left(q^{2}\right)+O(q) Q\left(w_{i j}\right),
$$

where we only keep terms up to the linear order in momentum $\boldsymbol{q}$ and the strain tensor $w_{i j}$. We find

$$
\begin{aligned}
h_{\boldsymbol{q}}=\boldsymbol{q} & \left.\cdot \nabla_{\boldsymbol{k}} \mathcal{H}_{\boldsymbol{k}}\right|_{\boldsymbol{k}=\boldsymbol{k}_{W}}=-\hbar v_{F} q_{x} s^{y} \sigma^{z} \tau^{z}+\hbar v_{F} q_{y} s^{x} \sigma^{z} \\
& -\eta t_{d} q_{z} a \sin Q a \sigma^{x} \tau^{z}+t_{d} q_{z} a \cos Q a \sigma^{y} \tau^{z} .
\end{aligned}
$$

If we assume constant strain $\nabla_{\boldsymbol{r}} w_{i j}=0$, the spectrum of Eq. 15 can be found

$$
\begin{aligned}
& \tilde{\epsilon}_{\boldsymbol{k}, \pm}^{2}=\frac{\hbar^{2} v_{F}^{2}}{a^{2}}\left[\left(q_{x}+\eta w_{31} \sin a Q\right)^{2}+\left(q_{y}+\eta w_{32} \sin a Q\right)^{2}\right] \\
& +\left[m \pm m \mp \frac{\eta t_{s} t_{d} \sin a Q}{m}\left(q_{z} a+\eta w_{33} \frac{m^{2}-|\Delta|^{2}}{t_{s} t_{d} \sin a Q}\right)\right]^{2}
\end{aligned}
$$

From this we can extract the magnitudes of the Fermi velocity components

$$
\left(v_{x}, v_{y}, v_{z}\right)=\left(v_{F}, v_{F},\left|\frac{t_{s} t_{d} \sin a Q}{m}\right|\right) .
$$

In addition we observe that the effect of constant distortion is to translate the Weyl points in the momentum 


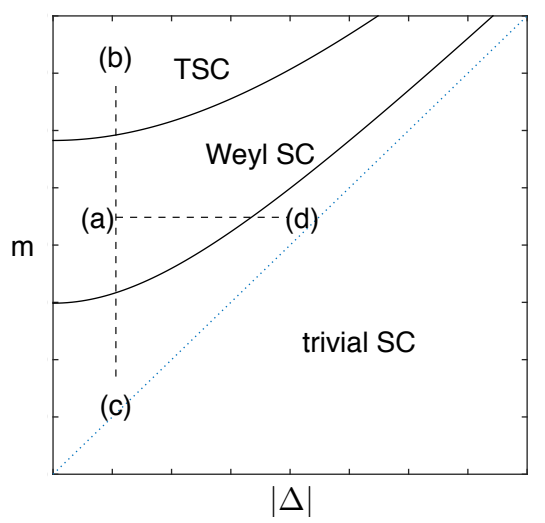

FIG. 4: Phase diagram of the Weyl superconductor described by Hamiltonian (2) in terms of $(m,|\Delta|)$ with labels (a)-(d) correspond to spectra shown in Fig. 3(a)-(d). The two black curves mark the phase boundaries given in Eq. 10 and Eq. (11). The dotted line indicates the asymptote for the two phase boundaries.

space by

$$
e \mathcal{A}=-\frac{\eta \hbar}{a}\left(w_{31} \sin Q a, w_{32} \sin Q a, w_{33} \frac{m^{2}-|\Delta|^{2}}{t_{s} t_{d} \sin Q a}\right)
$$

Clearly, the vector $\mathcal{A}$ can be understood as the gauge potential of a strain-induced chiral magnetic field.

To gain additional insight into this result, one can project $h_{\boldsymbol{q}}+\delta \mathcal{H}_{\boldsymbol{k}_{W}}$ onto the Hilbert space spanned by 4 zero-energy eigenvectors of $\mathcal{H}_{\boldsymbol{k}_{W}}$. The resulting Hamiltonian has massless Dirac form with the strain entering via minimal substitution. The strain induced chiral gauge potential can then be extracted and is consistent with Eq. (19). Details about this approach are given in Appendix A.

In most cases we expect $Q a \ll 1$. In this limit the $z$ component of $\mathcal{A}$ given in Eq. (19) scales as $1 / a Q$ but $x(y)$ components scale as $a Q$. Thus only $\mathcal{A}_{z}$ will be considered in the following.

We obtained $\mathcal{A}$ above by assuming constant distortion $w_{i j}$. However, we are interested in the bending deformation as shown in Fig. 1(b) where $w_{i j}$ is obviously spacedependent. We argue that even in this case the strain effect can be treated as a gauge field expressed in Eq. (19), as long as it varies slowly on the lattice scale. To support our argument, we first find the expression for $w_{33}$ when the system is bent and then numerically calculate the spectra of the tight-binding Hamiltonian with this distortion.

The bending deformation can be characterized by an small angle $\theta=a / \rho$ where $\rho$ is the radius of the circular bend. The lattice constant of the outermost $y$-direction layer is then $a+\delta a$ with $\delta a=\frac{1}{2} \bar{l}_{y} a \theta$. Here $\bar{l}_{y}$ is the number of layers in $y$-direction. Thus

$$
\rho=\frac{\bar{l}_{y} a}{2 \delta a / a}=\frac{\bar{l}_{y} a}{\varepsilon}
$$

with $\varepsilon=2 \delta a / a$ the bending parameter used in the numerics. Now if we consider a generic $y$-direction layer, its lattice constant will change by $\delta a(y)=\left(y-\bar{l}_{y} a / 2\right) \theta$. Then for a point with coordinate $z$ on this layer, its $z$ direction displacement is $u_{3}=\frac{z}{a}\left(y-\bar{l}_{y} a / 2\right) \frac{2 \delta a}{\bar{l}_{y} a}$. Thus,

$$
w_{33}=\frac{\partial u_{3}}{\partial z}=\left(y-\bar{l}_{y} a / 2\right) \frac{2 \delta a}{a^{2} \bar{l}_{y}}=\left(y-\bar{l}_{y} a / 2\right) \frac{\varepsilon}{\bar{l}_{y} a} .
$$

Therefore, we expect a pseudo-magnetic field

$$
\boldsymbol{b}=\partial_{y} \mathcal{A}_{z} \hat{x}=-\eta \frac{\hbar}{e a^{2}} \frac{m^{2}-|\Delta|^{2}}{t_{s} t_{d} \sin Q a} \frac{\varepsilon}{\bar{l}_{y}} \hat{x}
$$

Such pseudo-magnetic field will give rise to Dirac-Landau levels at energies

$$
\tilde{\epsilon}_{n}(\boldsymbol{k})= \pm \sqrt{\hbar^{2} v_{x}^{2} k_{x}^{2}+2 n\left|\frac{e b}{\hbar} \hbar v_{y} \hbar v_{z}\right|}
$$

for all integers $n \neq 0$ and $\tilde{\epsilon}_{0}\left(k_{x}\right)=-\hbar v_{F} k_{x}$ as the zeroth Landau levels for both valleys. In view of Eq. 22 we get

$$
\tilde{\epsilon}_{n}(\boldsymbol{k})= \pm \sqrt{\hbar^{2} v_{x}^{2} k_{x}^{2}+2 n \frac{\varepsilon}{\bar{l}_{y}} \frac{m^{2}-|\Delta|^{2}}{m} \frac{\hbar v_{y}}{a} .}
$$

We have numerically checked Eq. (24) by applying hopping substitutions Eq. (12) in the multilayer Hamiltonian Eq. 11 with $\bar{l}_{y}=150$, as summarized in Fig. 5 . Indeed we observe that the Dirac-Landau levels in Eq. (23) capture the features of the low-energy spectrum of the Weyl superconductor multilayer. For comparison we also plot the spectrum and DOS for the unstrained system and show the result in Fig. 5 (a,b).

For the sake of completeness we in addition calculate the spectrum of our model Weyl superconductor in the presence of the magnetic field $\mathbf{B} \| \hat{z}$ and the Abrikosov vortex lattice. This is summarized in Appendix D. We find that all bands become completely flat Landau levels in the $x-y$ plane. The zeroth Landau level, which is associated with Weyl nodes before $\mathbf{B}$ is switched on, is still linearly dispersive along the $z$-direction in the vicinity of nodes. In contrast, it is well known that the magnetic field does not lead to flat Landau levels in $d_{x^{2}-y^{2}}$ superconductors. This is because the spatially varying supercurrent in the vortex lattice strongly scatters the Bogoliubov quasiparticles [11. The difference between the $d_{x^{2}-y^{2}}$ and 3D Weyl superconductors has been recently elucidated in Ref. 38. with which our results are in accord. In short, the zeroth Landau level cannot be scattered by vortices due to the protection of Weyl node chirality, which is a topologically nontrivial and unique feature of the Weyl superconductor. 

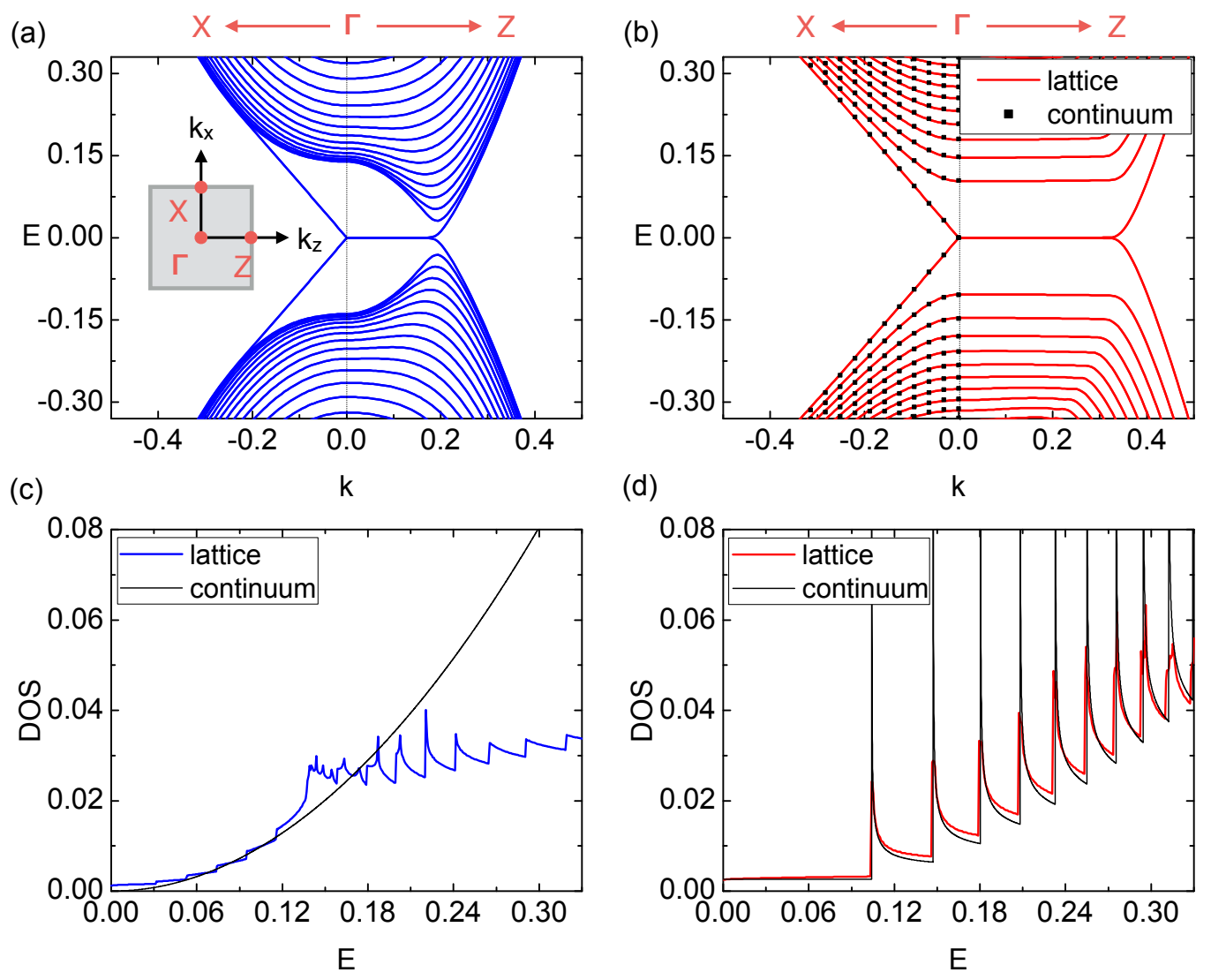

FIG. 5: Energy spectra and DOS for our Weyl superconductor with open boundaries and $\bar{l}_{y}=150$ along the $y$-direction and periodic along $x$ and $z$. (a) The spectrum of undeformed system; the flat band at zero energy is the Fermi arc. (b) The spectrum of a bent Weyl superconductor as shown in Fig. 1(b) with $\varepsilon=8 \%$ corresponding to a pseudo-magnetic field $b=10.45 \mathrm{~T}$. For both (a) and (b) the spectrum is plotted along $X-\bar{\Gamma}-Z$ as shown in the inset. For comparison, energy levels Eq. (24) are overlain as black dots. (c) DOS of the unstrained sample (blue curve) compared to the ideal $\sim E^{2}$ DOS expected for a massless Dirac fermion in continuum (black parabola). (d) DOS of the strained system (red curve) compared to DOS calculated for ideal Dirac-Landau levels with $b=10.45 \mathrm{~T}$.

\section{LONGITUDINAL THERMAL CONDUCTIVITY}

In Section III] we found that a bending deformation results in Dirac-Landau levels for Bogoliubov quasiparticles, which unlike those in Weyl semimetals, are charge neutral on average. Therefore, Shubnikov-de Haas quantum oscillation discussed in Ref. 33] cannot be observed in Weyl superconductors. But Bogoliubov quasiparticles do carry heat, making thermal transport measurements, such as the thermal Hall effect, possible [39 41].

We will evaluate in this Section the longitudinal thermal conductivity $\kappa_{x x}$ as a function of pseudo-magnetic field and show that $\kappa_{x x}$ exhibits oscillations periodic in $1 / b$. In our analysis above the chemical potential $\mu$ of TI layers was assumed to lie at the Weyl point. Here we will tune $\mu$ away from this "neutrality point" to obtain a finite size Fermi surface in order to observe quantum oscillation in $\kappa_{x x}$. For $\mu \neq 0$ Eq. (3) will be modified by an extra term $\delta \mathcal{H}_{\boldsymbol{k}}=-\mu \tau^{z}$. This term lifts the 2-fold degeneracy in the spectrum Eq. (4). Perturbative analysis detailed in Appendix B shows that the resulting spectrum can be well approximated by split Dirac-Landau levels of the form

$$
E_{n}(\boldsymbol{k})= \pm \sqrt{\hbar^{2} v_{x}^{2} k_{x}^{2}+2 n\left|\frac{e b}{\hbar} \hbar v_{y} \hbar v_{z}\right|} \pm \mu
$$

illustrated in Fig. 6(b). The corresponding DOS at the chemical potential is

$$
\begin{aligned}
D(0)= & \frac{L_{y} L_{z}}{2 \pi l_{B}^{2}} \sum_{n} L_{x} \sum_{ \pm} \int \frac{d k_{x}}{2 \pi} \delta\left(E_{n}(\boldsymbol{k})\right), \\
= & \frac{V}{2 \pi^{2} l_{B}^{2}} \frac{2}{\hbar v_{x}} \sum_{n} \sqrt{\frac{\mu^{2}}{\mu^{2}-2 n\left|\frac{e b}{\hbar} \hbar v_{y} \hbar v_{z}\right|}}
\end{aligned}
$$

where $l_{B}=\sqrt{\hbar / e b}$ is the magnetic length. The thermal conductivity can be computed using the Boltzmann 

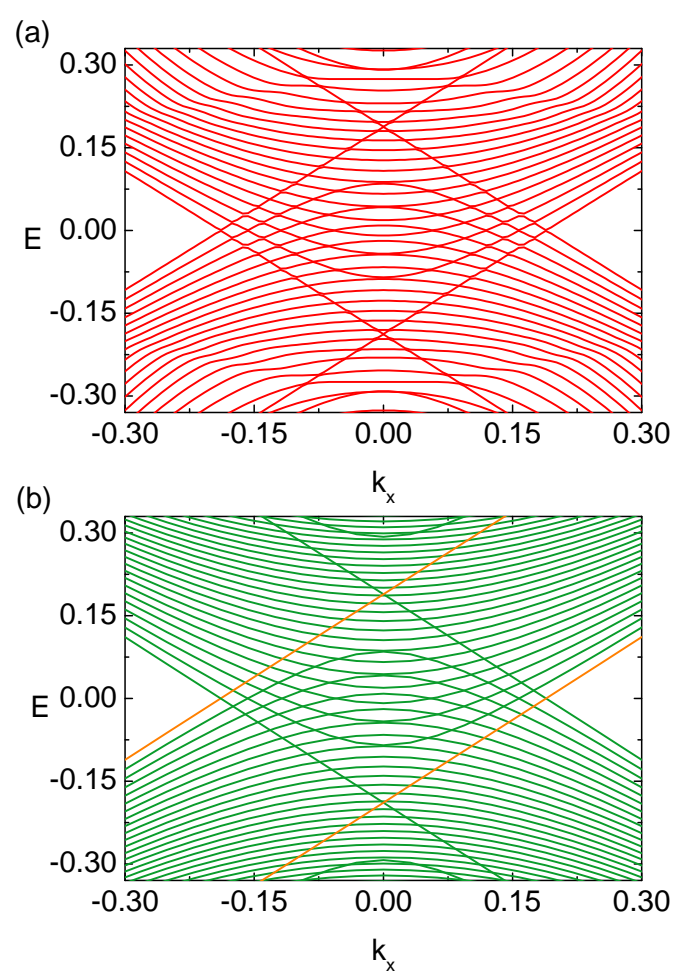

FIG. 6: Energy spectrum of the Weyl SC with the chemical potential of the TI layers tuned away from the surface Dirac points to $\mu=0.19$. (a) Quasiparticle spectrum calculated from the lattice model Eq. 13. It is worth noting that only the left moving chiral mode is due to the Landau quantization while the other is a surface mode. (b) Quasiparticle spectrum predicted by Eq. 25. To compare with the first panel, the chiral modes (orange lines) due to the surface states have been added manually.

equation approach 42,44 and reads

$$
\boldsymbol{\kappa}=\frac{1}{T} \sum_{n} \sum_{\boldsymbol{k}} E_{n}^{2}(\boldsymbol{k}) \tau_{n}(\boldsymbol{k}) \boldsymbol{v}_{n}(\boldsymbol{k}) \boldsymbol{v}_{n}(\boldsymbol{k})\left(-\frac{\partial f}{\partial E_{n}}\right)
$$

where $E_{n}(\boldsymbol{k})$ is the quasiparticle energy, $\boldsymbol{v}_{n}(\boldsymbol{k})=$ $\frac{1}{\hbar} \nabla_{\boldsymbol{k}} E_{n}(\boldsymbol{k})$ is the associated velocity, $\tau_{n}(\boldsymbol{k})$ is the corresponding scattering time, and $f\left(E_{n}\right)=\left(e^{E_{n} / k_{B} T}+1\right)^{-1}$. For our purposes, it is useful to rewrite the thermal conductivity (27) in the low- $T$ limit through the Sommerfeld expansion as explained in Appendix C. To leading order one obtains

$$
\boldsymbol{\kappa}=\frac{\pi^{2} k_{B}^{2} T}{3} \sum_{n} \sum_{\boldsymbol{k}} \tau_{n}(\boldsymbol{k}) \delta\left(E_{n}(\boldsymbol{k})\right) \boldsymbol{v}_{n}(\boldsymbol{k}) \boldsymbol{v}_{n}(\boldsymbol{k}) .
$$

The scattering rate can be approximated by Fermi's golden rule (see Appendix C) as

$$
\left.\tau_{n}^{-1}(\boldsymbol{k})\right|_{E_{n}(\boldsymbol{k})=0}=\frac{2 \pi}{\hbar} n_{\mathrm{imp}} C_{\mathrm{imp}} D(0)
$$

where $n_{\text {imp }}$ and $C_{\text {imp }}$ denote the impurity concentration and the scattering potential strength, respectively. The longitudinal thermal conductivity then becomes

$$
\kappa_{x x}(b)=\kappa_{x x}(0) \frac{\sum_{n} \sqrt{\frac{\mu^{2}-2 n\left|\frac{e b}{\hbar} \hbar v_{y} \hbar v_{z}\right|}{\mu^{2}}}}{\sum_{n} \sqrt{\frac{\mu^{2}}{\mu^{2}-2 n\left|\frac{e b}{\hbar} \hbar v_{y} \hbar v_{z}\right|}}}
$$

with the zero-field thermal conductivity

$$
\kappa_{x x}(0)=\frac{\pi^{2} k_{B}^{2} T}{3} \frac{v_{x}^{2}}{\frac{2 \pi}{\hbar} n_{\mathrm{imp}} C_{\mathrm{imp}}} .
$$

Fig. 7 shows our results for DOS and $\kappa_{x x}(b)$ calculated from the approximate analytical formulas Eqs. 26) and (30), and based on the full lattice calculation using Eqs. (26) and (28). They agree well and exhibit pronounced quantum oscillations periodic in $1 / b$.

We note that due to Landau quantization, thermal conductivity quantum oscillations in the $x$-direction are most pronounced, while in the other directions, quantum oscillations are expected to be weaker. Based on our results for the electronic structure in Fig. 5(b), the $z$-direction drift velocity of Bogoliubov quasiparticles is nonzero only at the edges of bands $\left(k_{z} a \sim 0.3\right)$. In contrast, the $x$ direction drift velocity is nonzero for almost all momenta. Therefore, $\kappa_{z z}$ should be small and its quantum oscillations are weaker than those in $\kappa_{x x}$. In y-direction, quasiparticle wave functions are Gaussian-localized with the characteristic decay length $\sqrt{\hbar v_{y} / e b v_{z}} \sim l_{B}$ and localization centers $2 \pi \nu l_{B}^{2} / L_{z}$ with $\nu=1,2, \cdots, L_{z} / a$. The localization makes transport difficult unless the localization center is pumped across the system when $b$ varies. Therefore, we do not expect pronounced quantum oscillations along the $y$-direction.

It is also worth noting that quasiparticle thermal conductivity can be obscured in real materials by phonons because phonons also carry heat. For temperature $T \ll$ $T_{c}$, the thermal conductivity of acoustic phonons follows Debye $T^{3}$ law $\kappa_{p h}^{A} \sim T^{3}$. The less dominant optical phonon thermal conductivity is $\kappa_{p h}^{O} \sim \frac{1}{T^{2}} \exp \left(-\frac{1}{T}\right)$. At low temperatures both will be overwhelmed by quasiparticle contribution. At higher temperatures, the phonon contribution $\kappa_{p h}=\kappa_{p h}^{A}+\kappa_{p h}^{O}$ can dominate over the quasiparticle thermal conductivity but quantum oscillations should remain visible. We do not expect $\kappa_{p h}$ to show quantum oscillations because phonons are bosonic excitations.

\section{CONCLUSIONS}

In this work, we studied a minimal model for a Weyl superconductor with a single pair of Weyl points based on the Meng-Balents layer construction. A MajoranaFermi arc appears and connects the two Weyl points if a pair of boundaries are open. This arc can be understood as being formed of two counter propagating chiral 


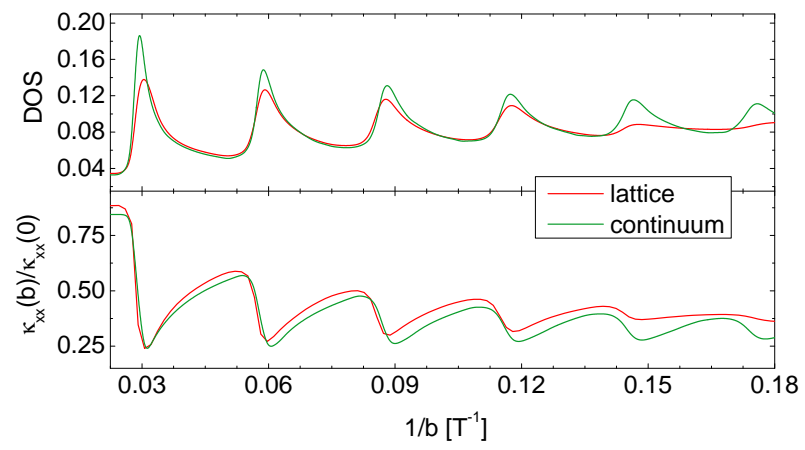

FIG. 7: Strain-induced quantum oscillation in a Weyl semimatal. The upper panel shows oscillations in DOS as a function of inverse strain strength expressed as $1 / b$ at zero-energy. The lower panel shows oscillations in the longitudinal quasiparticle thermal conductivity $\kappa_{x x}$. To simulate the effect of disorder, all data are broadened by convolving in energy with a Lorentzian with width $\delta_{\epsilon}=1.67 \times 10^{-3}$.

Majorana modes at the edge of an effective topological $p_{x}+i p_{y} \mathrm{SC}$ that results from fixing one component of the momentum in the 3D Hamiltonian describing the original Weyl SC. The phase diagram shows that the Weyl SC phase appears intermediate between a fully gapped trivial superconducting phase and a topological superconductor phase. These features elucidate similarities between Weyl superconductors and Weyl semimetals.

In the low-energy sector of the theory, we showed that elastic strain acts as a chiral gauge potential incorporated in the Weyl Hamiltonian through standard minimal substitution. Therefore, similar to graphene and Weyl semimetals, strain can mimic the effect of real physical magnetic field in the Weyl superconductor. One important difference is that the strain-induced pseudomagnetic field is not subject to the Meisner effect. Remarkably, this fact allows the pseudo-magnetic field to Landau quantize the spectrum of Bogoliubov quasiparticles instead of being expelled from the sample or creating Abrikosov vortex lattice as would be the case for physical magnetic field $B$.

Landau quantization generates pronounced quantum oscillations that can be observed by quasiparticle spectroscopy and by longitudinal thermal conductivity. These quantum oscillations occur deep in the superconducting state and are thus fundamentaly different from various theoretical proposals and experimental results that pertain to mixed and normal states of superconductors.

To experimentally test our proposal we require a Weyl superconductor. Such can be in principle artificially engineered through the Meng-Balents construction or can occur naturally in a suitable crystalline solid. Currently, there are roughly twenty different nodal superconductors known to science 45 . One of the most promising candidates may be $\mathrm{Cu}_{x} \mathrm{Bi}_{2} \mathrm{Se}_{3}[23,46$. Nuclear magnetic res- onance experiments 47] revealed broken spin rotational symmetry in $\mathrm{Cu}_{x} \mathrm{Bi}_{2} \mathrm{Se}_{3}$, suggesting the superconducting gap structure to be either $\Delta_{4 x}$ where the nodes appear due to the protection of mirror symmetry or $\Delta_{4 y}$ where small gaps (or nodes) are expected. Recent experimental results on the specific heat [48] of $\mathrm{Cu}_{x} \mathrm{Bi}_{2} \mathrm{Se}_{3}$ are consistent with nematic superconductivity and favor $\Delta_{4 y}$ pairing structure. Unfortunately, the gap minima and nodes cannot be straightforwardly differentiated based on the reported specific heat data alone. However, symmetry and energetic considerations [49, 50] suggest gap minima in nematic superconductivity. Another promising candidate is $\mathrm{Nb}_{x} \mathrm{Bi}_{2} \mathrm{Se}_{3}$, whose low temperature penetration depth exhibits quadratic temperature dependence characteristic of linearly dispersing point nodes in three dimensions [51]. This is consistent with $\mathrm{Nb}_{x} \mathrm{Bi}_{2} \mathrm{Se}_{3}$ being a Weyl superconductor. Although it is too early to draw a firm conclusion regarding the pairing state of the candidate materials, the existing experimental data give hope that $\mathrm{Cu}_{x} \mathrm{Bi}_{2} \mathrm{Se}_{3}$ and $\mathrm{Nb}_{x} \mathrm{Bi}_{2} \mathrm{Se}_{3}$ could eventually be identified as 3D Dirac or Weyl superconductors.

The second requirement is that the candidate material be sufficiently flexible to allow a few percent elastic deformation in order to generate a sufficiently strong pseudo-magnetic field. The candidate material should be prepared in a nanoscale thin film geometry in order to maximize its flexibility. To the best of our knowledge, detailed data on the mechanical properties of $\mathrm{Cu}_{x} \mathrm{Bi}_{2} \mathrm{Se}_{3}$ is lacking and further experimental work is needed to determine whether or not this could be a suitable material.

There are several future directions that might be interesting to pursue based on our current work. The first is to test other properties associated with pseudo-Landau levels. Recent work on the fractional Josephson effect in strained 2D graphene superconductor 52 motivates the interest in studying a similar effect in one dimension higher using strained Weyl superconductor. The second lies in the study of the chiral anomaly, chiral magnetic effect, and gravitational anomaly with straininduced gauge field.

\section{Acknowledgments}

The authors are indebted to T. Meng, A. Tsuruta, Y. Yanase, T. Matsushita, Z. Shi, and A. Chen for illuminating discussions. TL thanks JREP program under Topo-Q network offered by the "Topological Materials Science" project for the support at the initial stage of this work. SF is supported by the Grant-in-Aid for Scientific Research from MEXT of Japan [Grants No. 17K05517, No. 25220711, and No. 15H05852 (KAKENHI on Innovative Areas Topological Materials Science)]. This work is supported by NSERC, CIfAR and Max Planck - UBC Centre for Quantum Materials. 
[1] D. Shoenberg, Magnetic Oscillations in Metals (Cambridge University Press, Cambridge, 1984).

[2] J. G. Checkelsky, Y. S. Hor, M.-H. Liu, D.-X. Qu, R. J. Cava, and N. Ong, Physical Review Letters 103, 246601 (2009).

[3] A. Taskin and Y. Ando, Physical Review B 80, 085303 (2009).

[4] K. Eto, Z. Ren, A. Taskin, K. Segawa, and Y. Ando, Physical Review B 81, 195309 (2010).

[5] J. G. Analytis, J.-H. Chu, Y. Chen, F. Corredor, R. D. McDonald, Z. Shen, and I. R. Fisher, Physical Review B 81, 205407 (2010).

[6] D.-X. Qu, Y. S. Hor, J. Xiong, R. J. Cava, and N. Ong, Science 329, 821 (2010).

[7] A. Potter, I. Kimchi, and A. Vishwanath, Nature communications 5, 5161 (2013).

[8] L. He, X. Hong, J. Dong, J. Pan, Z. Zhang, J. Zhang, and S. Li, Physical review letters 113, 246402 (2014).

[9] P. Moll, N. Nair, T. Helm, A. Potter, I. Kimchi, A. Vishwanath, and J. Analytis, Nature 535, 266 (2016).

[10] J. Xiong, S. Kushwaha, J. Krizan, T. Liang, R. Cava, and N. Ong, EPL (Europhysics Letters) 114, 27002 (2016).

[11] M. Franz and Z. Tešanović, Phys. Rev. Lett. 84, 554 (2000).

[12] O. Vafek, A. Melikyan, M. Franz, and Z. Tešanović, Phys. Rev. B 63, 134509 (2001).

[13] T. Liu and M. Franz, Physical Review B 92, 134519 (2015).

[14] N. Barišić, S. Badoux, M. K. Chan, C. Dorow, W. Tabis, B. Vignolle, G. Yu, J. Béard, X. Zhao, C. Proust, et al., Nature Physics 9, 761 (2013).

[15] N. Doiron-Leyraud, C. Proust, D. LeBoeuf, J. Levallois, J.-B. Bonnemaison, R. Liang, D. Bonn, W. Hardy, and L. Taillefer, Nature 447, 565 (2007).

[16] B. Ramshaw, B. Vignolle, J. Day, R. Liang, W. Hardy, C. Proust, and D. Bonn, Nature Physics 7, 234 (2011).

[17] D. LeBoeuf, N. Doiron-Leyraud, J. Levallois, R. Daou, J. Bonnemaison, N. Hussey, L. Balicas, B. Ramshaw, R. Liang, D. Bonn, et al., Nature 450, 533 (2007).

[18] G. Grissonnanche, O. Cyr-Choiniere, F. Laliberté, S. R. de Cotret, A. Juneau-Fecteau, S. Dufour-Beauséjour, N. Hussey, B. Vignolle, C. Proust, M. Sutherland, et al., NATURE 5 (2014).

[19] C. Jaudet, D. Vignolles, A. Audouard, J. Levallois, D. LeBoeuf, N. Doiron-Leyraud, B. Vignolle, M. Nardone, A. Zitouni, R. Liang, et al., Physical Review Letters 100, 187005 (2008).

[20] A. Alexandrov, Journal of Physics: Condensed Matter 20, 192202 (2008).

[21] S. Banerjee, S. Zhang, and M. Randeria, Nature communications 4, 1700 (2013).

[22] R. Corcoran, P. Meeson, Y. Onuki, P.-A. Probst, M. Springford, K. Takita, H. Harima, G. Guo, and B. Gyorffy, Journal of Physics: Condensed Matter 6, 4479 (1994).

[23] S. A. Yang, H. Pan, and F. Zhang, Physical review letters 113, 046401 (2014).

[24] T. Meng and L. Balents, Physical Review B 86, 054504 (2012).

[25] F. Guinea, M. I. Katsnelson, and A. K. Geim, Nat Phys 6, $30(2010)$.
[26] N. Levy, S. A. Burke, K. L. Meaker, M. Panlasigui, A. Zettl, F. Guinea, A. H. C. Neto, and M. F. Crommie, Science 329, 544 (2010).

[27] A. Cortijo, Y. Ferreirós, K. Landsteiner, and M. A. Vozmediano, Physical review letters 115, 177202 (2015).

[28] H. Sumiyoshi and S. Fujimoto, Physical review letters 116, 166601 (2016).

[29] D. Pikulin, A. Chen, and M. Franz, Physical Review X 6, 041021 (2016).

[30] A. G. Grushin, J. W. Venderbos, A. Vishwanath, and R. Ilan, Physical Review X 6, 041046 (2016).

[31] A. Cortijo, D. Kharzeev, K. Landsteiner, and M. A. Vozmediano, Physical Review B 94, 241405 (2016).

[32] V. Arjona, E. V. Castro, and M. A. Vozmediano, arXiv preprint arXiv:1703.05399 (2017).

[33] T. Liu, D. Pikulin, and M. Franz, Physical Review B 95, 041201 (2017).

[34] G. Massarelli, G. Wachtel, J. Y. T. Wei, and A. Paramekanti, preprint arXiv:1707.07683 (2017).

[35] E. M. Nica and M. Franz, preprint arXiv:1709.01158 (2017).

[36] A. Burkov and L. Balents, Physical review letters 107, 127205 (2011).

[37] H. Shapourian, T. L. Hughes, and S. Ryu, Physical Review B 92, 165131 (2015).

[38] M. Pacholski, C. Beenakker, and I. Adagideli, preprint arXiv:1710.00719 (2017).

[39] J. M. Murray and O. Vafek, Physical Review B 92, 134520 (2015).

[40] H. Sumiyoshi and S. Fujimoto, Journal of the Physical Society of Japan 82, 023602 (2013).

[41] Z. Wang, X.-L. Qi, and S.-C. Zhang, Physical Review B 84, 014527 (2011).

[42] N. Ong, K. Krishana, Y. Zhang, and Z. Xu, in Physics and Chemistry of Transition Metal Oxides: Proceedings of the 20th Taniguchi Symposium, Kashikojima, Japan, May 25-29, 1998 (Springer Verlag, 1999), vol. 125, p. 202.

[43] J. Bardeen, G. Rickayzen, and L. Tewordt, Physical Review 113, 982 (1959).

[44] L. Tewordt, Physical Review 128, 12 (1962).

[45] A. P. Schnyder and P. M. Brydon, Journal of Physics: Condensed Matter 27, 243201 (2015).

[46] L. Fu and E. Berg, Physical review letters 105, 097001 (2010).

[47] K. Matano, M. Kriener, K. Segawa, Y. Ando, and G.-q. Zheng, Nature Physics 12, 852 (2016).

[48] S. Yonezawa, K. Tajiri, S. Nakata, Y. Nagai, Z. Wang, K. Segawa, Y. Ando, and Y. Maeno, Nature Physics 13, 123 (2016).

[49] L. Fu, Physical Review B 90, 100509 (2014).

[50] J. W. Venderbos, V. Kozii, and L. Fu, Physical Review B 94, 180504 (2016).

[51] M. Smylie, H. Claus, U. Welp, W.-K. Kwok, Y. Qiu, Y. S. Hor, and A. Snezhko, Physical Review B 94, 180510 (2016).

[52] S.-P. Lee, D. Nandi, F. Marsiglio, and J. Maciejko, Physical Review B 95, 174517 (2017). 


\section{Appendix A: Low-energy Weyl Hamiltonian}

In the main text Section III, we find that strain generally shifts the Weyl points and effectively works as a pseudo magnetic gauge potential. In this section, we will examine this from another point of view.

As the gauge field effect occurs in the low-energy sector, it will be sufficient to consider $\tilde{\mathcal{H}}_{\boldsymbol{k}_{W}+\boldsymbol{q}}$ defined in Eq. (15), namely

$$
\tilde{\mathcal{H}}_{\boldsymbol{k}_{W}+\boldsymbol{q}}=\mathcal{H}_{\boldsymbol{k}_{W}}+h_{\boldsymbol{q}}+\delta \mathcal{H}_{\boldsymbol{k}_{W}}
$$

where $\mathcal{H}_{\boldsymbol{k}_{W}}$ is the Hamiltonian at Weyl points $\boldsymbol{k}_{W}=$ $(0,0, \eta Q), h_{\boldsymbol{q}}$ is the linearized Hamiltonian, and $\delta \mathcal{H}_{\boldsymbol{k}_{W}}$ is induced by strain. To keep our derivation transparent, we perform the following substitution

$$
\begin{gathered}
\tilde{x}=\frac{\hbar v_{F}}{a}\left(q_{x} a+\eta w_{31} \sin Q a\right) \\
\tilde{y}=\frac{\hbar v_{F}}{a}\left(q_{y} a+\eta w_{32} \sin Q a\right) \\
\tilde{z}_{1}=-\eta t_{d} \sin Q a\left(q_{z} a+\eta w_{33} \frac{t_{s}+t_{d} \cos Q a}{t_{d} \sin Q a}\right) \\
\tilde{z}_{2}=t_{d} \cos Q a\left(q_{z} a-\eta w_{33} \tan Q a\right) \\
z_{1}=t_{s}+t_{d} \cos k_{z} a \\
z_{2}=t_{d} \sin k_{z} a
\end{gathered}
$$

With such definitions, we can write down the eigenvalues of $\mathcal{H}_{\boldsymbol{k}_{W}}$ as $\{2 m, 2 m, 0,0,0,0,-2 m,-2 m\}$. For real order parameter $\Delta \in \mathbb{R}$, the four eigenvectors corresponding to the Weyl points are

$$
\begin{aligned}
& \left|\phi_{1}\right\rangle=\frac{1}{\sqrt{2}}\left(\frac{z_{1}-i z_{2}}{m}, 0,-1,0,0,0,0, \frac{\Delta}{m}\right)^{T}, \\
& \left|\phi_{2}\right\rangle=\frac{1}{\sqrt{2}}\left(-\frac{\Delta}{m}, 0,0,0,0,1, \frac{z_{1}+i z_{2}}{m}\right)^{T}, \\
& \left|\phi_{3}\right\rangle=\frac{1}{\sqrt{2}}\left(0, \frac{-z_{1}+i z_{2}}{m}, 0,-1,0,0, \frac{\Delta}{m}, 0\right)^{T}, \\
& \left|\phi_{4}\right\rangle=\frac{1}{\sqrt{2}}\left(0,-\frac{\Delta}{m}, 0,0,1,0, \frac{-z_{1}-i z_{2}}{m}, 0\right)^{T} .
\end{aligned}
$$

We then project

$$
h_{\boldsymbol{q}}+\delta \mathcal{H}_{\boldsymbol{k}_{W}}=-\tilde{x} s^{y} \sigma^{z} \tau^{z}+\tilde{y} s^{x} \sigma^{z}+\tilde{z}_{1} \sigma^{x} \tau^{z}+\tilde{z}_{2} \sigma^{y} \tau^{z}
$$

onto the four-dimensional Hilbert space spanned by $\left|\phi_{i=1,2,3,4}\right\rangle$. We get

$$
\begin{aligned}
& \left(h_{\boldsymbol{q}}+\delta \mathcal{H}_{\boldsymbol{k}_{W}}\right)_{\phi}= \\
& \left(\begin{array}{cccc}
-\frac{z_{1} \tilde{z}_{1}+z_{2} \tilde{z}_{2}}{m} & 0 & -i \tilde{x}-\tilde{y} & 0 \\
0 & -\frac{z_{1} \tilde{z}_{1}+z_{2} \tilde{z}_{2}}{m} & 0 & i \tilde{x}+\tilde{y} \\
i \tilde{x}-\tilde{y} & 0 & \frac{z_{1} \tilde{z}_{1}+z_{2} \tilde{z}_{2}}{m} & 0 \\
0 & -i \tilde{x}+\tilde{y} & 0 & \frac{z_{1} \tilde{z}_{1}+z_{2} \tilde{z}_{2}}{m}
\end{array}\right)
\end{aligned}
$$

The projected $4 \times 4$ matrix Hamiltonian can be written in terms of standard Dirac matrices which we express as a tensor product of Pauli matrices $\alpha$ and $\beta$ as

$$
\begin{array}{r}
\left(h_{\boldsymbol{q}}+\delta \mathcal{H}_{\boldsymbol{k}_{W}}\right)_{\phi}=\frac{\hbar v_{F}}{a}\left(q_{x} a+\eta w_{31} \sin Q a\right) \alpha^{z} \beta^{y} \\
-\frac{\hbar v_{F}}{a}\left(q_{y} a+\eta w_{32} \sin Q a\right) \alpha^{z} \beta^{x} \\
\frac{\eta t_{s} t_{d} \sin a Q}{m}\left(q_{z} a+\eta w_{33} \frac{m^{2}-\Delta^{2}}{t_{s} t_{d} \sin a Q}\right) \beta^{z}
\end{array}
$$

From here, one can read off the strain-induced a gauge field

$$
\mathcal{A}=-\frac{\eta \hbar}{e a}\left(w_{31} \sin Q a, w_{32} \sin Q a, w_{33} \frac{m^{2}-\Delta^{2}}{t_{s} t_{d} \sin Q a}\right)
$$

consistent with Eq. 19 in the main text.

\section{Appendix B: Weyl superconductor with chemical potential}

In the main text, we studied a Weyl superconductor multilayer with the chemical potential of the TI set to zero. In this case, the Fermi surface of the Weyl SC shrinks to two points (Weyl nodes). To observe quantum oscillation, we need a finite size Fermi surface, and this can be achieved by switching on the chemical potential of the TI layers. In this section, we will show that with nonzero chemical potential, implemented in Eq. (3) by adding an extra term $-\mu \tau^{z}$, the 2-fold degeneracy of the spectrum Eq. (4) will lift. One copy of the quasiparticle spectrum will move up by $\mu$ and the other will move down by $\mu$ creating a Fermi surface with a nonzero volume.

To see this, we write down the following Hamiltonian

$$
\mathcal{H}_{k}-\mu \tau^{z}=H_{0}+V
$$

where

$$
\begin{gathered}
H_{0}=m_{0} s^{z} \tau^{z}+z_{1} \sigma^{x} \tau^{z}+z_{2} \sigma^{y} \tau^{z}-\Delta s^{y} \tau^{y} \\
V=-\mu \tau^{z}+y s^{x} \sigma^{z}-x s^{y} \sigma^{z} \tau^{z}
\end{gathered}
$$

Again, for simplicity we assume real $\Delta$ and define

$$
\begin{gathered}
m_{0}=m-4 m^{\prime}+2 m^{\prime} \cos k_{x} a+2 m^{\prime} \cos k_{y} a \\
x=\frac{\hbar v_{F}}{a} \sin k_{x} \\
y=\frac{\hbar v_{F}}{a} \sin k_{y}
\end{gathered}
$$

Based on the parameter values we have chosen in the main text, $\Delta$ is of the same order as $z_{2}$, but is one order of magnitude smaller than $z_{1}$ and $m_{0}$. We set chemical potential $\mu$ to be an order of magnitude smaller than $\Delta$. This allows us to treat $V$ as a perturbation to $H_{0}$, whose low-energy eigenvectors can be easily resolved as 


$$
\begin{aligned}
\left|\phi_{D_{1}, 1}\right\rangle & =\frac{1}{\sqrt{2}}\left(\frac{z_{1}-i z_{2}}{\sqrt{z_{1}^{2}+z_{2}^{2}+\Delta^{2}}}, 0,-1,0,0,0,0, \frac{\Delta}{\sqrt{z_{1}^{2}+z_{2}^{2}+\Delta^{2}}}\right)^{T}, \\
\left|\phi_{D_{1}, 2}\right\rangle & =\frac{1}{\sqrt{2}}\left(\frac{-\Delta}{\sqrt{z_{1}^{2}+z_{2}^{2}+\Delta^{2}}}, 0,0,0,0,1,0, \frac{z_{1}+i z_{2}}{\sqrt{z_{1}^{2}+z_{2}^{2}+\Delta^{2}}}\right)^{T}, \\
\left|\phi_{D_{2}, 1}\right\rangle & =\frac{1}{\sqrt{2}}\left(0, \frac{-z_{1}+i z_{2}}{\sqrt{z_{1}^{2}+z_{2}^{2}+\Delta^{2}}}, 0,-1,0,0, \frac{\Delta}{\sqrt{z_{1}^{2}+z_{2}^{2}+\Delta^{2}}}, 0\right)^{T}, \\
\left|\phi_{D_{2}, 2}\right\rangle & =\frac{1}{\sqrt{2}}\left(0, \frac{-\Delta}{\sqrt{z_{1}^{2}+z_{2}^{2}+\Delta^{2}}}, 0,0,1,0, \frac{-z_{1}-i z_{2}}{\sqrt{z_{1}^{2}+z_{2}^{2}+\Delta^{2}}}, 0\right)^{T} .
\end{aligned}
$$

These correspond to the degenerate subspace $D_{1}$ with eigenvalue

$$
E_{D_{1}, 1(2)}^{(0)}=m_{0}-\sqrt{z_{1}^{2}+z_{2}^{2}+\Delta^{2}}
$$

and $D_{2}$ with eigenvalue

$$
E_{D_{2}, 1(2)}^{(0)}=\sqrt{z_{1}^{2}+z_{2}^{2}+\Delta^{2}}-m_{0}
$$

We then project the perturbation $V$ to the degenerate subspaces $D_{1}$ and $D_{2}$, respectively. In a compact form, it reads

$$
V_{D_{i}}=\left(\begin{array}{cc}
\left\langle\phi_{D_{i}, 1}|V| \phi_{D_{i}, 1}\right\rangle & \left\langle\phi_{D_{i}, 1}|V| \phi_{D_{i}, 2}\right\rangle \\
\left\langle\phi_{D_{i}, 2}|V| \phi_{D_{i}, 1}\right\rangle & \left\langle\phi_{D_{i}, 2}|V| \phi_{D_{i}, 2}\right\rangle
\end{array}\right)
$$

where $i=1,2$. Individually, we can write $V_{D_{i}}$ in terms of the Pauli matrix $\boldsymbol{\nu}$

$$
\begin{aligned}
& V_{D_{1}}=d_{x} \nu^{x}+d_{y} \nu^{y}+d_{z} \nu^{z} \\
& V_{D_{2}}=-d_{x} \nu^{x}-d_{y} \nu^{y}+d_{z} \nu^{z}
\end{aligned}
$$

where

$$
\begin{aligned}
& d_{x}=\frac{z_{1} \Delta}{z_{1}^{2}+z_{2}^{2}+\Delta^{2}} \mu \\
& d_{y}=-\frac{z_{2} \Delta}{z_{1}^{2}+z_{2}^{2}+\Delta^{2}} \mu \\
& d_{z}=-\frac{z_{1}^{2}+z_{2}^{2}}{z_{1}^{2}+z_{2}^{2}+\Delta^{2}} \mu
\end{aligned}
$$

Matrices $V_{D_{1}}$ and $V_{D_{2}}$ can be diagonalized through unitary transformations

$$
U_{D_{i}}^{-1} V_{D_{i}} U_{D_{i}}=\operatorname{diag}(d,-d) \quad i=1,2
$$

where

$$
d=\sqrt{\frac{z_{1}^{2}+z_{2}^{2}}{z_{1}^{2}+z_{2}^{2}+\Delta^{2}}} \mu \mu
$$

because for our purpose $\Delta^{2} \ll z_{1}^{2}+z_{2}^{2}$. The transforma- tion matrices are

$$
\begin{aligned}
& U_{D_{1}}=\left(\begin{array}{cc}
\sqrt{\frac{d+d_{z}}{2 d}} \frac{d_{x}-i d_{y}}{\sqrt{d_{x}^{2}+d_{y}^{2}}} & -\sqrt{\frac{d-d_{z}}{2 d}} \\
\sqrt{\frac{d-d_{z}}{2 d}} & \sqrt{\frac{d+d_{z}}{2 d}} \frac{d_{x}+i d_{y}}{\sqrt{d_{x}^{2}+d_{y}^{2}}}
\end{array}\right), \\
& U_{D_{2}}=\left(\begin{array}{cc}
\sqrt{\frac{d+d_{z}}{2 d}} \frac{-d_{x}+i d_{y}}{\sqrt{d_{x}^{2}+d_{y}^{2}}} & -\sqrt{\frac{d-d_{z}}{2 d}} \\
\sqrt{\frac{d-d_{z}}{2 d}} & \sqrt{\frac{d+d_{z}}{2 d}} \frac{-d_{x}-i d_{y}}{\sqrt{d_{x}^{2}+d_{y}^{2}}}
\end{array}\right) .
\end{aligned}
$$

We can immediately write down the first order correction to energy

$$
E_{D_{1}, 1(2)}^{(1)}=E_{D_{2}, 1(2)}^{(1)}= \pm d \approx \pm \mu
$$

Because the 2-fold degeneracy is lifted, the zeroth order eigenvectors are now uniquely determined

$$
\begin{aligned}
& \left(\left|\tilde{\phi}_{D_{1}, 1}\right\rangle,\left|\tilde{\phi}_{D_{1}, 2}\right\rangle\right)=\left(\left|\phi_{D_{1}, 1}\right\rangle,\left|\phi_{D_{1}, 2}\right\rangle\right) U_{D_{1}}, \\
& \left(\left|\tilde{\phi}_{D_{2}, 1}\right\rangle,\left|\tilde{\phi}_{D_{2}, 2}\right\rangle\right)=\left(\left|\phi_{D_{2}, 1}\right\rangle,\left|\phi_{D_{2}, 2}\right\rangle\right) U_{D_{2}}
\end{aligned}
$$

We may now calculate the second order correction to the energy

$$
\begin{gathered}
E_{D_{1}, 1(2)}^{(2)}=\sum_{\alpha \in D_{2}} \frac{\left|\left\langle\tilde{\phi}_{\alpha}|V| \tilde{\phi}_{D_{1}, 1(2)}\right\rangle\right|^{2}}{E_{D_{1}}-E_{\alpha}} \\
=\frac{x^{2}+y^{2}}{2\left(m_{0}-\sqrt{z_{1}^{2}+z_{2}^{2}+\Delta^{2}}\right)} \\
E_{D_{2}, 1(2)}^{(2)}=\sum_{\alpha \in D_{1}} \frac{\left|\left\langle\tilde{\phi}_{\alpha}|V| \tilde{\phi}_{D_{2}, 1(2)}\right\rangle\right|^{2}}{E_{D_{2}}-E_{\alpha}} \\
=-\frac{x^{2}+y^{2}}{2\left(m_{0}-\sqrt{z_{1}^{2}+z_{2}^{2}+\Delta^{2}}\right)}
\end{gathered}
$$

where we ignore the contribution from high-energy sector, if any. This is because for high energies $E_{\alpha}=$ $\pm\left(m_{0}+\sqrt{z_{1}^{2}+z_{2}^{2}+\Delta^{2}}\right)$ the denominator in the second order correction is either $\pm 2 m_{0}$ or $\pm 2 \sqrt{z_{1}^{2}+z_{2}^{2}+\Delta^{2}}$, whose magnitude is much larger than that of $\pm 2\left(m_{0}-\right.$ 
$\sqrt{z_{1}^{2}+z_{2}^{2}+\Delta^{2}}$ ) and thus are less important. Combining all the corrections, we can estimate the quasiparticle energy at nozero $\mu$ as

$$
\begin{aligned}
& E_{D_{1}, 1(2)} \approx \sqrt{x^{2}+y^{2}+\left(m_{0}-\sqrt{z_{1}^{2}+z_{2}^{2}+\Delta^{2}}\right)^{2}} \pm \mu, \\
& E_{D_{2}, 1(2)} \approx-\sqrt{x^{2}+y^{2}+\left(m_{0}-\sqrt{z_{1}^{2}+z_{2}^{2}+\Delta^{2}}\right)^{2}} \pm \mu .
\end{aligned}
$$

We observe that to leading order the spectrum of Weyl superconductor multilayer is now biased. The original 2fold degeneracy in Eq. (4) has been lifted. One copy of spectrum moves up while the other copy moves down. As a result, the strain induced pseudo Landau levels will also be biased as in Eq. 25

\section{Appendix C: Thermal conductivity}

In this section, we will derive the expression for the thermal conductivity given in Eq. (28) of main text. Our starting point is Eq. (27). In order to calculate $\boldsymbol{\kappa}$ analytically, we introduce the auxiliary tensor

$$
\boldsymbol{\sigma}(\epsilon)=\sum_{n} \sum_{\boldsymbol{k}} \tau_{n}(\boldsymbol{k}) \delta\left(\epsilon-E_{n}(\boldsymbol{k})\right) \boldsymbol{v}_{n}(\boldsymbol{k}) \boldsymbol{v}_{n}(\boldsymbol{k}),
$$

which may be understood as a thermal analogue of the usual conductivity tensor. It is easy to see that

$$
\boldsymbol{\kappa}=\frac{1}{T} \int_{-\infty}^{+\infty} d \epsilon \epsilon^{2} \boldsymbol{\sigma}(\epsilon)\left(-\frac{\partial f}{\partial \epsilon}\right)
$$

We further define an auxiliary function

$$
K(\epsilon)=\epsilon^{2} \boldsymbol{\sigma}(\epsilon)
$$

through which the thermal conductivity can be written as

$$
\kappa=\left.\frac{1}{T} \int_{-\infty}^{+\infty} d \epsilon \sum_{s=1}^{\infty} \frac{1}{s !} \frac{d^{s} K}{d \epsilon^{s}}\right|_{0} \epsilon^{s}\left(-\frac{\partial f}{\partial \epsilon}\right)
$$

Note that $\frac{\partial f}{\partial \epsilon}$ is an even function of $\epsilon$. Therefore, we only need to consider even $s$. The thermal conductivity is further simplified as

$$
\kappa=\left.\frac{1}{T} \sum_{s=1}^{\infty} \int_{-\infty}^{+\infty} d \epsilon \frac{\left(k_{B} T\right)^{2 s}}{(2 s) !}\left(\frac{\epsilon}{k_{B} T}\right)^{2 s}\left(-\frac{\partial f}{\partial \epsilon}\right) \frac{d^{2 s} K}{d \epsilon^{2 s}}\right|_{0}
$$

Define $x=\frac{\epsilon}{k_{B} T}$ and

$$
\begin{aligned}
a_{s}=\int_{-\infty}^{+\infty} d x & \frac{x^{2 s}}{(2 s) !}\left(-\frac{d}{d x} \frac{1}{e^{x}+1}\right) \\
= & \frac{2}{\Gamma(2 s)} \int_{0}^{+\infty} d x \frac{x^{2 s-1}}{e^{x}+1} \\
& =2 \eta(2 s)=2\left(1-2^{1-2 s}\right) \zeta(2 s)
\end{aligned}
$$

where $\Gamma(s)=\int_{0}^{+\infty} d x \frac{x^{s-1}}{e^{x}}, \eta(s)=\int_{0}^{+\infty} d x \frac{x^{s-1}}{e^{x}+1}$, and $\zeta(s)=\int_{0}^{+\infty} d x \frac{x^{s-1}}{e^{x}-1}$ are Gamma function, Dirichlet eta function, and Riemann zeta function, respectively. The thermal conductivity now reads

$$
\boldsymbol{\kappa}=\left.\frac{1}{T} \sum_{s=1}^{\infty} 2\left(1-2^{1-2 s}\right) \zeta(2 s) \frac{d^{2 s} K}{d \epsilon^{2 s}}\right|_{0}\left(k_{B} T\right)^{2 s}
$$

For low temperatures $k_{B} T \ll \mu$, we keep only the $s=1$ term and use $\zeta(2)=\frac{\pi^{2}}{6}$ to get

$$
\begin{aligned}
\boldsymbol{\kappa}=\frac{1}{T} \frac{\pi^{2} k_{B}^{2} T^{2}}{3} \boldsymbol{\sigma}(0)= \\
\quad \frac{\pi^{2} k_{B}^{2} T}{3} \sum_{n} \sum_{\boldsymbol{k}} \tau_{n}(\boldsymbol{k}) \delta\left(E_{n}(\boldsymbol{k})\right) \boldsymbol{v}_{n}(\boldsymbol{k}) \boldsymbol{v}_{n}(\boldsymbol{k})
\end{aligned}
$$

This relation can be regarded as the Wiedemann-Franz law for Bogoliubov quasiparticles.

The scattering time can be determined by Fermi's golden rule

$$
\begin{aligned}
\frac{1}{\tau_{n}(\boldsymbol{k})}=\frac{2 \pi}{\hbar} \sum_{n^{\prime}} \sum_{\boldsymbol{k}^{\prime}} \mid\left\langle n^{\prime} \boldsymbol{k}^{\prime}\right| & \left.V(\boldsymbol{r})_{\mathrm{imp}} \tau^{z}|n \boldsymbol{k}\rangle\right|^{2} \\
& \times \delta\left(E_{n}(\boldsymbol{k})-E_{n^{\prime}}\left(\boldsymbol{k}^{\prime}\right)\right)
\end{aligned}
$$

where $|n \boldsymbol{k}\rangle$ is the eigenvector of chemical potential biased Weyl superconductor under strain, characterized by the Hamiltonian $H-\mu \tau^{z}$. As discussed in Appendix B, when $\mu^{2} \ll \Delta^{2} \ll t_{s}^{2}+t_{d}^{2}+2 t_{s} t_{d} \cos k_{z} a$, we can use perturbative calculation to write down the Schrödinger equations for Weyl superconductor with TI layer chemical potential $\mu \neq 0$ and $\mu=0$, respectively.

$$
\begin{aligned}
\left(H-\mu \tau^{z}\right)\left|n \boldsymbol{k}^{0}\right\rangle & \approx \tilde{\epsilon}_{n}(\boldsymbol{k})\left|n \boldsymbol{k}^{0}\right\rangle \pm \mu\left|n \boldsymbol{k}^{0}\right\rangle, \\
H\left|n \boldsymbol{k}^{0}\right\rangle & =\tilde{\epsilon}_{n}(\boldsymbol{k})\left|n \boldsymbol{k}^{0}\right\rangle,
\end{aligned}
$$

where $\tilde{\epsilon}_{n}(\boldsymbol{k})$ is determined by Eq. 24$)$ and $\left|n \boldsymbol{k}^{0}\right\rangle$ is the exact eigenvector of $H$ and the zeroth order eigenvector of $H-\mu \tau^{z}$. If apply $\left\langle n^{\prime} \boldsymbol{k}^{\prime 0}\right|$ to Eq. (C10) and Eq. (C11) and subtract, we get

$$
\left\langle n^{\prime} \boldsymbol{k}^{\prime 0}\left|\tau^{z}\right| n \boldsymbol{k}^{0}\right\rangle \approx \pm\left\langle n^{\prime} \boldsymbol{k}^{\prime 0} \mid n \boldsymbol{k}^{0}\right\rangle
$$

then we can approximate $\tau_{n}(\boldsymbol{k})$ by

$$
\begin{array}{r}
\frac{1}{\tau_{n}(\boldsymbol{k})} \approx \frac{2 \pi}{\hbar} \sum_{n^{\prime}} \sum_{\boldsymbol{k}^{\prime}}\left|\left\langle n^{\prime} \boldsymbol{k}^{\prime 0}\left|V(\boldsymbol{r})_{\mathrm{imp}}\right| n \boldsymbol{k}^{0}\right\rangle\right|^{2} \\
\times \delta\left(E_{n}(\boldsymbol{k})-E_{n^{\prime}}\left(\boldsymbol{k}^{\prime}\right)\right)
\end{array}
$$

The righthand side is the same as the scattering rate of a Weyl semimetal 33 . with electronic structure characterized by $E_{n}(\boldsymbol{k})$. Therefore, the scattering rate in a Weyl superconductor should also be the same which leads to Eq. 290 in the main text. 


\section{Appendix D: Vortex lattice}

In this section we study Weyl superconductors under real magnetic field $\boldsymbol{B}$ and compare the results to Section III] in the main text. Due to the Meissner effect, $\boldsymbol{B}$ field is known to generate quasiparticle Bloch waves rather than Dirac-Landau levels in two-dimensional nodal SC, such as those with a $d$-wave symmetry of the gap function [11, 12. It is however not known how this result translates to three-dimensional Weyl SC.

We consider magnetic field along $z$-direction, so that $k_{z}$ remains a good quantum number. Thus, the system can, in principle, stay gapless. To study the vortex lattice, we write Eq. (1) as

$$
H=\frac{1}{2} \sum_{k} \Psi_{r}^{\dagger} \mathcal{H}_{\boldsymbol{r}} \Psi_{\boldsymbol{r}}=\frac{1}{2} \sum_{\boldsymbol{k}} \Psi_{\boldsymbol{r}}^{\dagger}\left(\begin{array}{cc}
\mathcal{H}_{r}^{11} & \mathcal{H}_{\boldsymbol{r}}^{12} \\
\mathcal{H}_{\boldsymbol{r}}^{21} & \mathcal{H}_{\boldsymbol{r}}^{22}
\end{array}\right) \Psi_{\boldsymbol{r}}
$$

with the real space basis to be written as $\Psi_{r}=$ $\left(c_{\boldsymbol{r}, \uparrow, 1}, c_{\boldsymbol{r}, \downarrow, 1}, c_{\boldsymbol{r}, \uparrow, 2}, c_{\boldsymbol{r}, \downarrow, 2}, c_{\boldsymbol{r}, \uparrow, 1}^{\dagger}, c_{\boldsymbol{r}, \downarrow, 1}^{\dagger}, c_{\boldsymbol{r}, \uparrow, 2}^{\dagger}, c_{\boldsymbol{r}, \downarrow, 2}^{\dagger}\right)^{T}$ and the blocks are defined as

$$
\begin{aligned}
& \mathcal{H}_{\boldsymbol{r}}^{11}=\left(\begin{array}{cccc}
m-4 b+b \sum_{\delta} \hat{s}_{\delta} & -i \frac{\hbar v_{F}}{2 a} \sum_{\delta} \hat{\eta}_{\delta}^{*} & t_{s}+t_{d} e^{-i k_{z} a} & 0 \\
-i \frac{\hbar v_{F}}{2 a} \sum_{\delta} \hat{\eta}_{\delta} & -m+4 b-b \sum_{\delta} \hat{s}_{\delta} & 0 & t_{s}+t_{d} e^{-i k_{z} a} \\
t_{s}+t_{d} e^{i k_{z} a} & 0 & m-4 b+b \sum_{\delta} \hat{s}_{\delta} & i \frac{\hbar v_{F}}{2 a} \sum_{\delta} \hat{\eta}_{\delta}^{*} \\
0 & t_{s}+t_{d} e^{i k_{z} a} & i \frac{\hbar v_{F}}{2 a} \sum_{\delta} \hat{\eta}_{\delta} & -m+4 b-b \sum_{\delta} \hat{s}_{\delta}
\end{array}\right) \\
& \mathcal{H}_{\boldsymbol{r}}^{22}=\left(\begin{array}{cccc}
-m+4 b-b \sum_{\delta} \hat{s}_{\delta} & -i \frac{\hbar v_{F}}{2 a} \sum_{\delta} \hat{\eta}_{\delta} & -t_{s}-t_{d} e^{-i k_{z} a} & 0 \\
-i \frac{\hbar v_{F}}{2 a} \sum_{\delta} \hat{\eta}_{\delta}^{*} & m-4 b+b \sum_{\delta} \hat{s}_{\delta} & 0 & -t_{s}-t_{d} e^{-i k_{z} a} \\
-t_{s}-t_{d} e^{i k_{z} a} & 0 & -m+4 b-b \sum_{\delta} \hat{s}_{\delta} & i \frac{\hbar v_{F}}{2 a} \sum_{\delta} \hat{\eta}_{\delta} \\
0 & -t_{s}-t_{d} e^{i k_{z} a} & i \frac{\hbar v_{F}}{2 a} \sum_{\delta} \hat{\eta}_{\delta}^{*} & m-4 b+b \sum_{\delta} \hat{s}_{\delta}
\end{array}\right) \\
& \mathcal{H}_{r}^{12}=\left(\begin{array}{cccc}
0 & \Delta & 0 & 0 \\
-\Delta & 0 & 0 & 0 \\
0 & 0 & 0 & \Delta \\
0 & 0 & -\Delta & 0
\end{array}\right) \quad \mathcal{H}_{r}^{21}=\left(\begin{array}{cccc}
0 & -\Delta^{*} & 0 & 0 \\
\Delta^{*} & 0 & 0 & 0 \\
0 & 0 & 0 & -\Delta^{*} \\
0 & 0 & \Delta^{*} & 0
\end{array}\right)
\end{aligned}
$$

Here the shift operator is defined as

$$
\hat{s}_{\boldsymbol{\delta}} f(\boldsymbol{r})=f(\boldsymbol{r}+\boldsymbol{\delta}) \quad \boldsymbol{\delta}= \pm a \hat{x}, \pm a \hat{y}
$$

and

$$
\hat{\eta}_{\boldsymbol{\delta}}=\left\{\begin{array}{ll}
\mp i \hat{s}_{\boldsymbol{\delta}} & \text { if } \boldsymbol{\delta}= \pm a \hat{x} \\
\pm \hat{s}_{\boldsymbol{\delta}} & \text { if } \boldsymbol{\delta}= \pm a \hat{y}
\end{array} .\right.
$$

In order to model vortex lattice, the phase of $\Delta(\boldsymbol{r})=$ $\Delta_{0} e^{i \phi(\boldsymbol{r})}$ is taken to wind by $2 \pi$ around each vortex center. We solve the problem by performing a unitary transformation [1] in the Nambu space defined by

$$
U=\left(\begin{array}{cc}
e^{i \phi_{A}(\boldsymbol{r})} & 0 \\
0 & e^{-i \phi_{B}(\boldsymbol{r})}
\end{array}\right),
$$

where we have partitioned vortices into two sublattices A and B such that $\phi_{A}(\boldsymbol{r})+\phi_{B}(\boldsymbol{r})=\phi(\boldsymbol{r})$. This removes the phase winding from the off-diagonal part of the Hamiltonian and makes it periodic in real space with a unit cell depited in Fig. 8.

The eigenstates of the transformed Hamiltonian are Bloch waves 11,13 that read $\Phi_{n \boldsymbol{K}}(\boldsymbol{r})=$ $e^{i \boldsymbol{K} \cdot \boldsymbol{r}}\left[U_{n \boldsymbol{K}}(\boldsymbol{r}), V_{n \boldsymbol{K}}(\boldsymbol{r})\right]^{T}$ with crystal momentum $\boldsymbol{K}$ associated with the vortex lattice (Fig. 8). The BdG type Bloch Hamiltonian is $H_{\boldsymbol{K}}=e^{-i \boldsymbol{K} \cdot \boldsymbol{r}} U^{-1} H_{\boldsymbol{r}} U e^{i \boldsymbol{K} \cdot \boldsymbol{r}}$ with its 4 blocks $H_{\boldsymbol{K}}^{i j}=e^{-i \boldsymbol{K} \cdot \boldsymbol{r}} U^{-1} H_{\boldsymbol{r}}^{i j} U e^{i \boldsymbol{K} \cdot \boldsymbol{r}}$ defined as

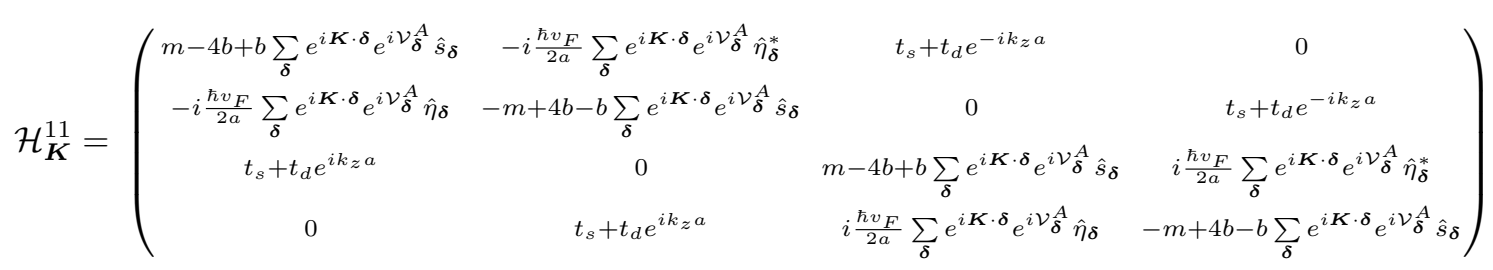




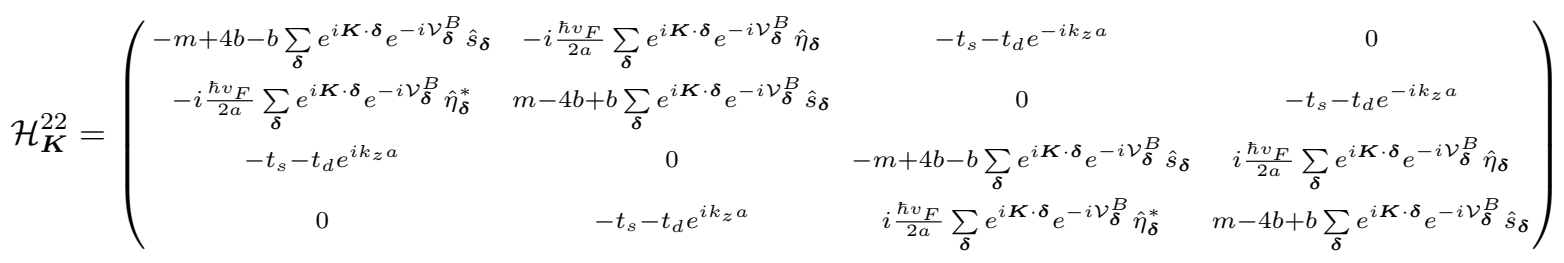

$$
\begin{aligned}
& \mathcal{H}_{\boldsymbol{K}}^{12}=\left(\begin{array}{cccc}
0 & \Delta & 0 & 0 \\
-\Delta & 0 & 0 & 0 \\
0 & 0 & 0 & \Delta \\
0 & 0 & -\Delta & 0
\end{array}\right) \quad \mathcal{H}_{\boldsymbol{K}}^{21}=\left(\begin{array}{cccc}
0 & -\Delta^{*} & 0 & 0 \\
\Delta^{*} & 0 & 0 & 0 \\
0 & 0 & 0 & -\Delta^{*} \\
0 & 0 & \Delta^{*} & 0
\end{array}\right)
\end{aligned}
$$

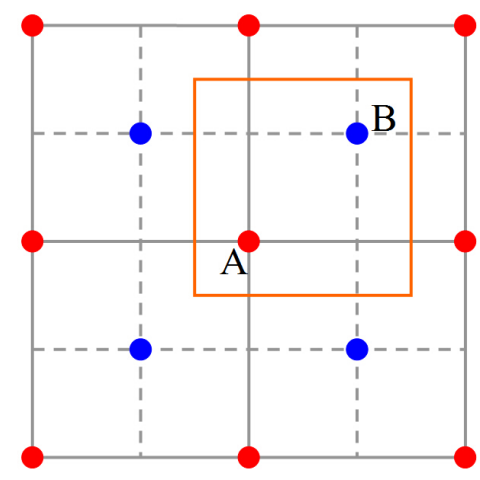

reciprocal lattice vectors $\boldsymbol{G}$ of the vortex lattice,

$$
\mathcal{V}_{\boldsymbol{\delta}}^{\mu}(\boldsymbol{r})=\frac{2 \pi}{L^{2}} \sum_{\boldsymbol{G}} \int_{\boldsymbol{r}}^{\boldsymbol{r}+\boldsymbol{\delta}} e^{i \boldsymbol{G} \cdot\left(\boldsymbol{r}-\boldsymbol{\delta}^{\mu}\right)} \frac{i \boldsymbol{G} \times \hat{z}}{G^{2}} \cdot d \boldsymbol{l}
$$

We apply Eq. (D13) to the real space Hamiltonian $H_{\boldsymbol{r}}$ and exactly diagonalize $H_{\boldsymbol{r}}$ for various vortex lattice configurations. The dispersions along the $k_{z}$-axis are summarized in Fig. 9(a-c). We observe that the Weyl points survive in as we change the A-B vortex distance within each unit cell. Surprisingly, the variation of the vortex positions barely changes the dispersion. Therefore, we conclude that the $k_{z}$ component of the Weyl dispersion is stable under magnetic field $B$ as long as vortices form a periodic lattice.

Dispersion in the $K_{x}-K_{y}$ plane however changes dramatically. In Fig. $9(\mathrm{~d}-\mathrm{f})$, we plot dispersions along $K_{x}$ for the vortex configurations used in panels (a-c). We see that the energy bands are reorganized into almost completely flat Dirac-Landau levels which are qualitatively similar to those reported by Ref. 38. For comparison we also indicate the expected energies $\sim \sqrt{n}$ of Dirac-Landau levels (orange curves) by matching to the $n=0,1$ bands. It is worth noting that the deviation of numerically calculated bands (green curves) from the ideal $\sqrt{n}$ sequence are due to the fact that Dirac-Landau levels exist only in the low-energy regime in the vicinity of the Weyl points. For our model, Lifshitz transition occurs at $E_{\text {Lif }}=0.138$. Therefore, we do not expect a perfect match to the $\sqrt{n}$ behavior beyond the lowest few energy levels.
Following the standard derivation [12 an expression for $\mathcal{V}_{\boldsymbol{\delta}}^{\mu}$ can be derived in terms of summation over the

$$
\boldsymbol{v}_{s}^{\mu}(\boldsymbol{r})=\frac{\hbar}{m}\left(\nabla \phi^{\mu}-\frac{e}{\hbar} \boldsymbol{A}(\boldsymbol{r})\right) \quad \mu=A, B .
$$


(a)

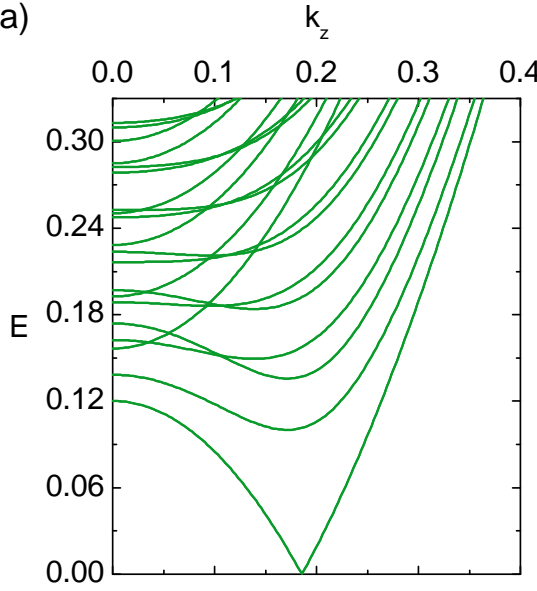

(d)

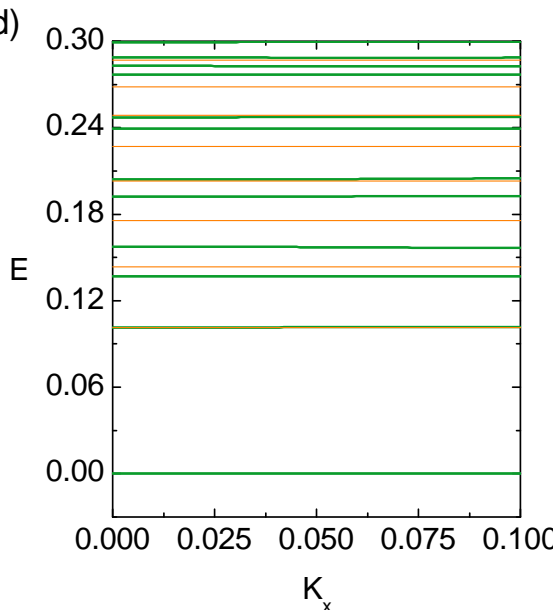

(b)

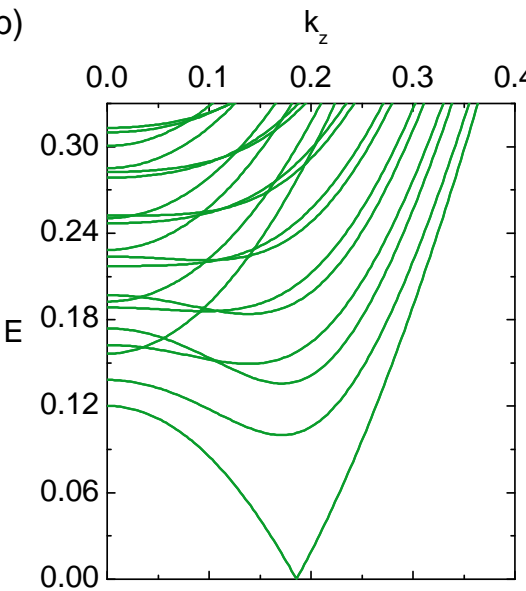

(e)

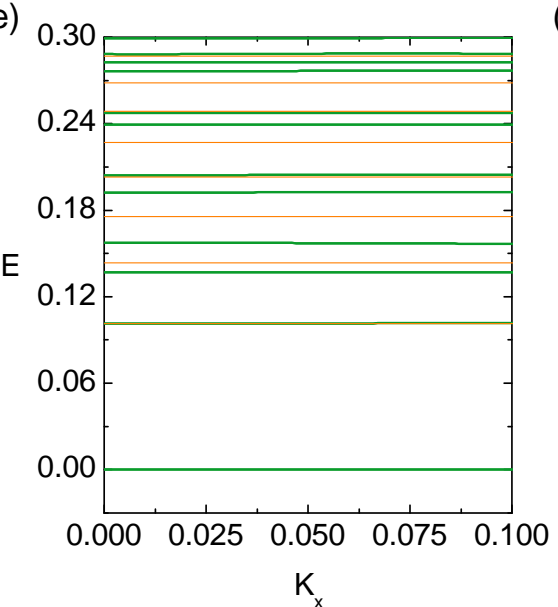

(c)

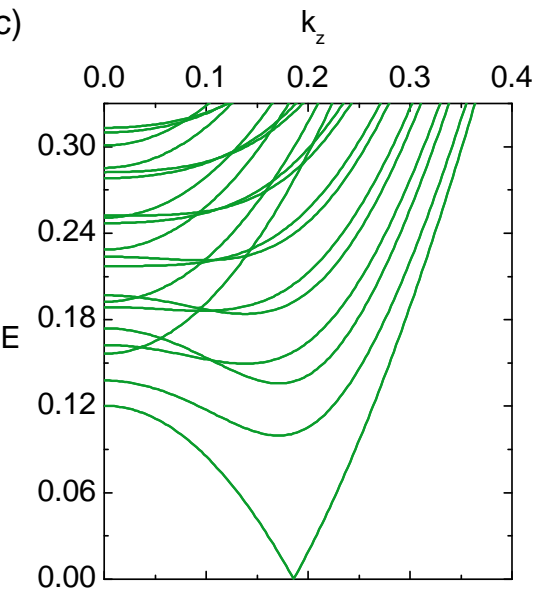

(f)

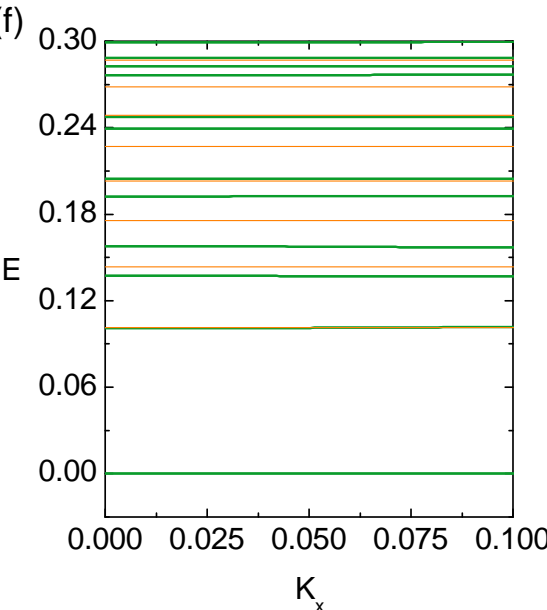

FIG. 9: Spectra of Weyl superconductor with vortex lattice. The size of magnetic unit cell is $L \times L=30 a \times 30 a$. The spacings between two vortices in the magnetic unit cell are (a) $\boldsymbol{d}=(15 a, 15 a)$ (b) $\boldsymbol{d}=(10 a, 10 a)(\mathrm{c}) \boldsymbol{d}=(5 a, 5 a)(\mathrm{d}) \boldsymbol{d}=(15 a, 15 a)(\mathrm{e})$ $\boldsymbol{d}=(10 a, 10 a)$ (f) $\boldsymbol{d}=(5 a, 5 a)$ The orange curves in panel (d)-(f) are analytical Dirac-Landau levels with $n=1$ band matched to the numerics. 